\title{
Magnetism in the spiral galaxy NGC 6946: magnetic arms, depolarization rings, dynamo modes, and helical fields
}

\author{
R. Beck \\ Max-Planck-Institut für Radioastronomie, Auf dem Hügel 69, 53121 Bonn, Germany \\ e-mail: rbeck@mpifr-bonn.mpg.de
}

Received 20 December 2006 / Accepted 8 May 2007

\begin{abstract}
Context. The spiral galaxy NGC 6946 hosts magnetic spiral arms, highly aligned magnetic fields between the gas/optical arms. Aims. The origin of the magnetic phenomena and their relation to the interstellar gas are investigated.

Methods. NGC 6946 was observed in total intensity and linear polarization in five radio bands between $3 \mathrm{~cm}$ and $21 \mathrm{~cm}$. Maps of spectral index, Faraday rotation and depolarization were derived.

Results. At the inner edge of the inner gas spiral arm the ordered magnetic field is only mildly compressed and turns smoothly, to become aligned along the gas arm. Hence the field is not shocked and is probably connected to the warm, diffuse gas. At larger radii, two bright magnetic arms between the optical arms are visible in polarized intensity. The field in the northern magnetic arm is almost totally aligned. Faraday rotation measures $(R M)$ in these arms are consistent with the superposition of two low azimuthal dynamo modes. Three more magnetic arms are discovered in the outer galaxy, located between $\mathrm{H}_{\mathrm{I}}$ arms. The $R M$ structure function confirms large-scale coherent fields. The observed anti-correlation between the field's pitch angles and the $R M$ values is a possible signature of helical fields. - Due to strong Faraday depolarization the galaxy is not transparent to polarized waves at $\lambda 18 \mathrm{~cm}$ and $\lambda 20 \mathrm{~cm}$. The large-scale asymmetry in depolarization with respect to the major axis may be another indication of large-scale helical fields. Three depolarization rings of almost zero polarization seen at $\lambda 20 \mathrm{~cm}$ are probably generated by differential Faraday rotation in $\mathrm{H}_{\mathrm{II}}$ complexes in NGC 6946 of 300-500 pc size. - In the gas/optical spiral arms, the total (mostly turbulent) magnetic field is amplified to $\simeq 20 \mu \mathrm{G}$. Its energy density is $\simeq 10$ times larger than that of the ionized gas and is similar to that of the turbulent gas motions in the inner galaxy. The magnetic energy exceeds that of the turbulent energy in the outer galaxy. All energy densities in NGC 6946 are about one order of magnitude larger than those in the Milky Way.

Conclusions. Density waves in the inner gaseous spiral arms mildly compress the field. Dynamo action probably generates the magnetic spiral arms. The magnetic field is dynamically important, interacts with the gas flow and possibly determines the properties of the gas spiral arms.
\end{abstract}

Key words. galaxies: spiral - galaxies: magnetic fields - galaxies: ISM - galaxies: individual: NGC 6946 radio continuum: galaxies - radio continuum: ISM

\section{Introduction}

Radio polarization observations revealed magnetic fields of considerable strength in all galaxies containing a significant amount of cold gas (see Beck 2005, for a review). Magnetic fields are probably important for star formation (Mac Low \& Klessen 2004; Heiles \& Crutcher 2005), especially the first generation of stars (Silk \& Langer 2006), and for the formation of spiral arms (Kim \& Ostriker 2002; Gómez \& Cox 2002), but their role is not yet understood.

NGC 6946 is a nearby grand-design spiral galaxy of Hubble type Scd at only $\approx 5.5 \mathrm{Mpc}$ distance (Tully 1988), so that $1^{\prime}$ corresponds to $1.6 \mathrm{kpc}$. With an optical diameter of about $20^{\prime}$ it is one of the largest galaxies in the sky. The spiral arms are massive, but without prominent dust lanes at their inner edges, indicating relatively weak density waves. No companion galaxy is known, and the small inner molecular bar can hardly excite density waves. The distribution of gas and star formation is asymmetric ("lopsided"), with one prominent spiral arm in the north-east.

NGC 6946 is bright in all spectral ranges and hence an ideal laboratory to test the interaction between the components of the interstellar medium. The radio-infrared correlation within galaxies was first studied there (Beck \& Golla 1988; Bicay et al. 1989). Linearly polarized radio continuum emission was first detected at $\lambda 2.8 \mathrm{~cm}$ with the Effelsberg telescope (Klein et al. 1982), followed by more sensitive Effelsberg observations at $\lambda 6.2 \mathrm{~cm}$ and $\lambda 2.8 \mathrm{~cm}$ (Harnett et al. 1989; Ehle \& Beck 1993) and observations with the VLA at $\lambda \lambda 18,20 \mathrm{~cm}$ (Beck 1991) and $\lambda 6 \mathrm{~cm}$ (Beck \& Hoernes 1996). The latter authors combined VLA and Effelsberg data and demonstrated that the missing small baselines of an interferometer reduce the large-scale emission not only in the Stokes parameter $I$, but also in $Q$ and $U$. Thus the combination of high-frequency data from synthesis and single-dish telescopes is also essential for polarization measurements.

The combined $\lambda 6.2 \mathrm{~cm}$ polarization map by Beck \& Hoernes (1996) revealed two surprisingly symmetric "magnetic spiral arms" located between the optical spiral arms. After subtraction of the diffuse unpolarized radio background the degree of polarization is $30-60 \%$. This means that the field in the magnetic arms is almost perfectly aligned, parallel to the adjacent spiral arms. The symmetry and alignment of the magnetic arms indicated that a new kind of physical phenomenon was 
discovered, with possibly major consequences for our understanding of galactic magnetic fields. Analysis of the magnetic arms in NGC 6946 with 1D wavelet functions by Frick et al. (2000) indicated that they are phase-shifted "images" of the optical arms. The cross-correlation technique using 2D isotropic wavelets demonstrated an anticorrelation between polarized intensity and gas density (Frick et al. 2001).

After the discovery of the magnetic arms in NGC 6946, several authors presented dynamo models with generation of magnetic arms (Moss 1998; Shukurov 1998; Rohde et al. 1999). Dynamo action is supported by the observational result that Faraday rotation measures in NGC 6946 are preferentially positive in the northern magnetic arm and negative in the southern arm, thus a large-scale field with a coherent direction exists (Ehle \& Beck 1993). As the northern side is nearer to us, the radial component of the spiral magnetic field points inwards, i.e. towards the galaxy's centre. A similar result was found for spiral fields in several other galaxies, but neither dynamo action nor large-scale primordial seed fields are able to explain this preference (Krause \& Beck 1998). An alternative interpretation of the magnetic arms as slow, phase-shifted MHD waves (Fan \& Lou 1996, 1997; Lou \& Fan 1998) was intriguing. However, slow MHD waves can exist only in the rigidly rotating part of a galaxy while the magnetic arms are mainly observed in the outer regions. Fundamental criticism was raised by Shukurov (2005) who claimed that slow MHD waves are unstable in three dimensions.

In spite of all efforts the phenomenon of magnetic arms is far from being understood. In this paper new multi-wavelength radio observations of NGC 6946 which were obtained during the last years are presented and discussed. The two main magnetic arms are confirmed at all wavelengths, but more magnetic arms exist. The almost perfect alignment of the magnetic field vectors along the arm structure suggests close interaction between the field and the gas flow.

\section{Observations and data reduction}

The main parameters of the radio continuum observations with the Very Large Array (VLA) operated by the $\mathrm{NRAO}^{1}$ and the Effelsberg telescope ${ }^{2}$ are given in Table 1. Reduction of the data was performed with the standard routines of the AIPS and NOD2 software packages, respectively. 3C138 and 3C286 were used for calibration of flux density and polarization angle. The secondary calibrator J2022+616 was measured during the VLA observation periods about once every 30 minutes and used for calibration of telescope gains and phases and for correction of instrumental polarization (routine PCAL). The accuracy of polarization angle calibration is better than $1^{\circ}$ for both telescopes. The level of instrumental polarization is less than $1 \%$ at the centre of the VLA primary beam, but increases to a few $\%$ at the half-power radius (Condon et al. 1998). However, the observations presented here are obtained from data observed over a wide range of parallactic angles so that the instrumental polarization is smoothed out. The instrumental polarization of the Effelsberg telescope emerges from the polarized sidelobes with $0.3-0.5 \%$ of the peak total intensity at the frequencies of the observations presented in this paper.

${ }^{1}$ The NRAO is a facility of the National Science Foundation operated under cooperative agreement by Associated Universities, Inc.

2 The Effelsberg 100-m telescope is operated by the Max-PlanckInstitut für Radioastronomie in Bonn on behalf of the Max-PlanckGesellschaft (MPG).
At $\lambda 3.6 \mathrm{~cm}$ the VLA maps in Stokes $I, Q$ and $U$ were combined (using LTESS) from three pointings centered at RA, Dec $(\mathrm{J} 2000)=20^{\mathrm{h}} 34^{\mathrm{m}} 55^{\mathrm{s}} .9,+60^{\circ} 10^{\prime} 24^{\prime \prime}$ (north), $20^{\mathrm{h}} 34^{\mathrm{m}} 31^{\mathrm{s}} .9,+60^{\circ} 09^{\prime} 13^{\prime \prime}$ (west) and $20^{\mathrm{h}} 34^{\mathrm{m}} 52^{\mathrm{s}} .1,+60^{\circ} 06^{\prime} 44^{\prime \prime}$ (south). Primary beam attenuation has been corrected for. Two sets of maps were obtained by robust and natural weighting of the $u v$ data, giving two different angular resolutions (8.'5 and $\left.12^{\prime \prime}\right)$. The maps at $12^{\prime \prime}$ resolution were merged with the Effelsberg data at $\lambda 2.8 \mathrm{~cm}$ by applying IMERG with an overlap in the baselines of the $u v$ data of 1000-1500 $\lambda$. (The minimum baseline of the VLA is about $850 \lambda$ while the maximum baseline of the Effelsberg telescope is about 3500 $\lambda$.) The small difference in wavelengths was corrected by assuming a mean spectral index of -0.7 , according to Ehle \& Beck (1993). The errors introduced by this assumption are negligible because the Effelsberg data are used to fill the largest missing structures only. The combined maps were smoothed to $15^{\prime \prime}(\simeq 400 \mathrm{pc})$ resolution to increase the signal-to-noise ratio.

At $\lambda 6.2 \mathrm{~cm}$ the VLA and Effelsberg maps were combined by Beck \& Hoernes (1996). For this paper the original resolution of the VLA maps of 12'. 5 (robust weighting) was increased to $15^{\prime \prime}$ in order to increase the signal-to-noise ratio. The galaxy was observed again more deeply at $\lambda 6.2 \mathrm{~cm}$ with the Effelsberg telescope to search for extended emission around the galaxy.

At $\lambda \lambda 18.0,20.5 \mathrm{~cm}$ the existing D-array data by Beck (1991) were combined with the more recent $\mathrm{C}$-array data in the $u v$ plane. Maps were generated at $15^{\prime \prime}$ resolution (robust weighting) and corrected for primary beam attenuation. Effelsberg data were observed at a similar wavelength. Flux integration of the VLA and Effelsberg maps gave similar values and hence no indication for missing large-scale structures in the VLA maps and no need for a combination.

The maps in Stokes $Q$ and $U$ were combined to maps of linearly polarized intensity $P I$ including the correction for positive bias due to noise (POLCO in AIPS and POLDEN in NOD2). Note that in the $P I$ maps the noise distribution is non-Gaussian, and the standard deviation underestimates the noise. Hence only rms noise values for the maps in Stokes $I, Q$ and $U$ are given in Table 1.

\section{Results}

\subsection{Total intensity}

The total radio intensities at $\lambda 6.2 \mathrm{~cm}$ and $\lambda 20.5 \mathrm{~cm}$ are shown in Figs. 1 and 2. The emission follows closely the distribution of IR emission, as has been discussed in previous papers (Frick et al. 2001; Walsh et al. 2002). According to Murphy et al. (2006) the radio image should be a smoothed version of the IR map due to diffusion of cosmic-ray electrons from the starforming regions. Figure 1 does not confirm this. The widths of several spiral arms, measured along slices across them, are identical in the radio and IR maps to within $1^{\prime \prime}$. The intensity of total synchrotron emission is proportional to $B_{\mathrm{t}}^{1-\alpha_{\mathrm{n}}}$ where $B_{\mathrm{t}}$ is the component of the total magnetic field in the sky plane and $\alpha_{\mathrm{n}}$ is the synchrotron spectral index $\left(I_{v} \propto v^{\alpha_{\mathrm{n}}}\right.$, with $\alpha_{\mathrm{n}} \simeq-1$, see below). In the equipartition case (see Sect. 3.3) $I_{v} \propto B_{\mathrm{t}}^{3-\alpha_{\mathrm{n}}}$. As a result of this strong dependence, the distribution of radio synchrotron emission in the disk of a spiral galaxy like NGC 6946 is dominated by the distribution of magnetic fields rather than by the propagation properties of cosmic rays, and the nonthermal part of the radio-infrared correlation is due to the coupling between magnetic fields and cold gas clouds (Niklas \& Beck 1997; Hoernes et al. 1998; Walsh et al. 2002). 
Table 1. Radio continuum observations of NGC 6946.

\begin{tabular}{|c|c|c|}
\hline & VLA & Effelsberg \\
\hline Frequency $(\mathrm{GHz})$ & 8.435 and 8.485 & 10.55 \\
\hline Wavelength $(\mathrm{cm})$ & 3.55 and 3.52 & 2.84 \\
\hline Configuration & $\mathrm{D}$ & \\
\hline Pointings & 3 & \\
\hline Observing dates & 1995 Apr. $13+20,1996$ July $21+23$ & 1990 June to 1991 Feb. \\
\hline Net observing time (h) & 26 & 60 \\
\hline Resolution of final maps & 8.5 and $15^{\prime \prime}$ & $68^{\prime \prime}$ \\
\hline Rms noise in $I ; Q$ and $U$ ( $\mu \mathrm{Jy} /$ beam area) & $10 ; 7$ and $15 ; 8$ & $450 ; 200$ \\
\hline Reference & & Ehle \& Beck (1993) \\
\hline Frequency $(\mathrm{GHz})$ & 4.835 and 4.885 & 4.85 \\
\hline Wavelength $(\mathrm{cm})$ & 6.20 and 6.14 & 6.18 \\
\hline Configuration & $\mathrm{D}$ & \\
\hline Pointings & 1 & \\
\hline Observing dates & 1991 Mar. 23 + Apr. 1 & 1997 Jan. to 1998 Sep. \\
\hline Net observing time $(\mathrm{h})$ & 10 & 18 \\
\hline Resolution of final maps & $15^{\prime \prime}$ & $147^{\prime \prime}$ \\
\hline Rms noise in $I ; Q$ and $U$ ( $\mu \mathrm{Jy} /$ beam area) & $22 ; 15$ & $500 ; 70$ \\
\hline Reference & Beck \& Hoernes (1996) & \\
\hline Frequency $(\mathrm{GHz})$ & & 2.675 \\
\hline Wavelength $(\mathrm{cm})$ & & 11.21 \\
\hline Observing dates & & 1999 Apr. to July \\
\hline Net observing time (h) & & 7 \\
\hline Resolution of final maps & & $300^{\prime \prime}$ \\
\hline Rms noise in $I ; Q$ and $U$ ( $\mu \mathrm{Jy} /$ beam area) & & $1000 ; 600$ \\
\hline Frequency $(\mathrm{GHz})$ & 1.465 and 1.665 & 1.400 \\
\hline Wavelength $(\mathrm{cm})$ & 20.46 and 18.01 & 21.41 \\
\hline Configuration & $\mathrm{D}+\mathrm{C}$ & \\
\hline Pointings & 1 & \\
\hline Observing date & 1988 July 26 + 1996 Apr. 4 & 1998 Sep. \\
\hline Net observing time $(\mathrm{h})$ & $12+11$ & 7 \\
\hline Resolution of final maps & $15^{\prime \prime}$ & $560^{\prime \prime}$ \\
\hline Rms noise in $I ; Q$ and $U$ ( $\mu \mathrm{Jy} /$ beam area) & $20 ; 12$ & $5000 ; 2000$ \\
\hline Reference & Beck (1991, D array) & \\
\hline
\end{tabular}
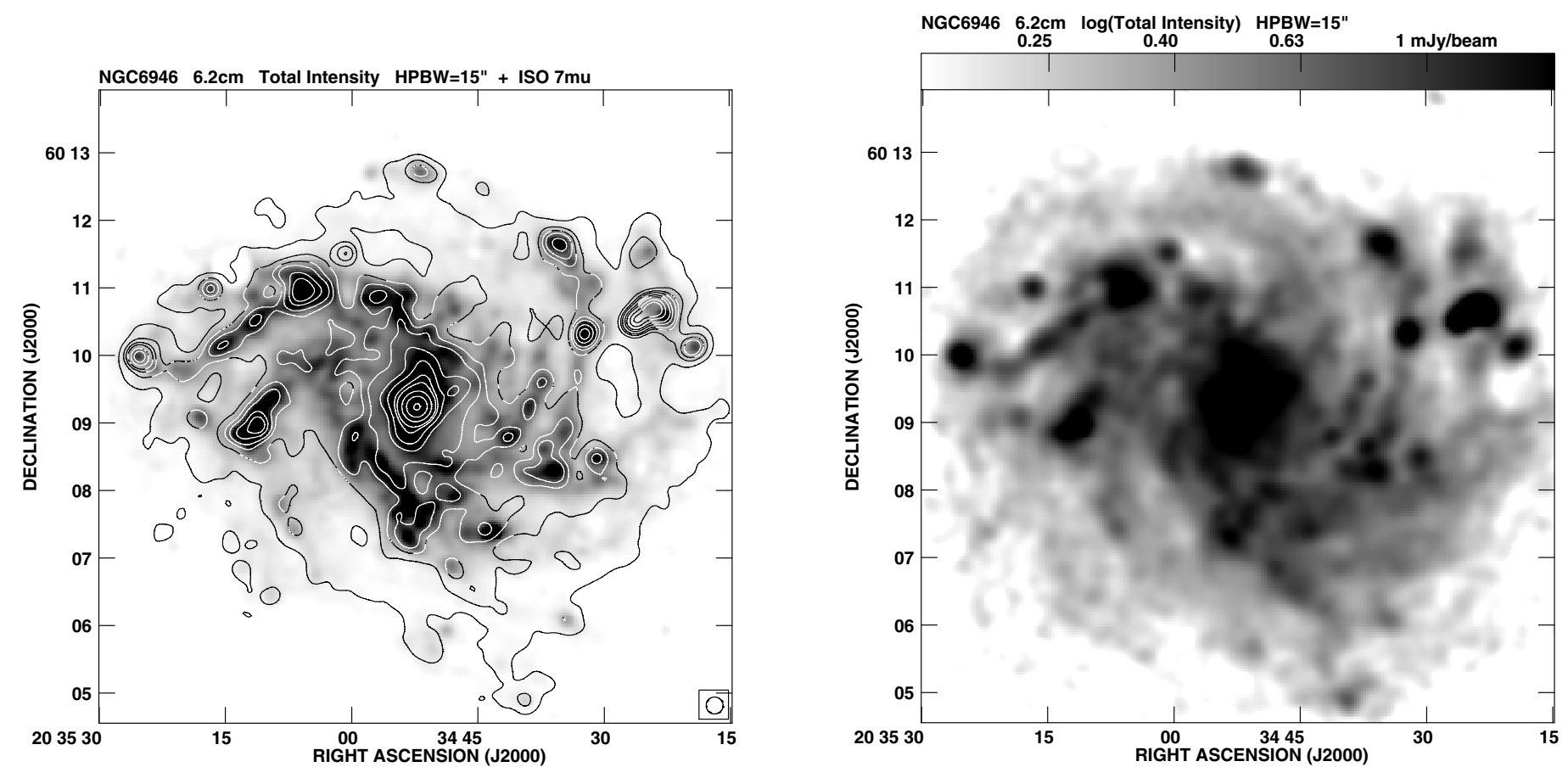

Fig. 1. Left: total intensity contours of NGC 6946 at $\lambda 6.2 \mathrm{~cm}$ at $15^{\prime \prime}$ resolution, combined from VLA and Effelsberg data. The contour intervals are $1,2,3,4,6,8,12,16,32,64,128 \times 300 \mu \mathrm{Jy} /$ beam area. The background greyscale image shows the $7 \mu \mathrm{m}$ IR emission measured with the ISO satellite with about 6 " resolution (Dale et al. 2000). Here and in the following maps, the beam size is shown in the bottom right or left corner of each panel. Right: logarithmic representation of the total intensity at $\lambda 6.2 \mathrm{~cm}$ and at $15^{\prime \prime}$ resolution. 

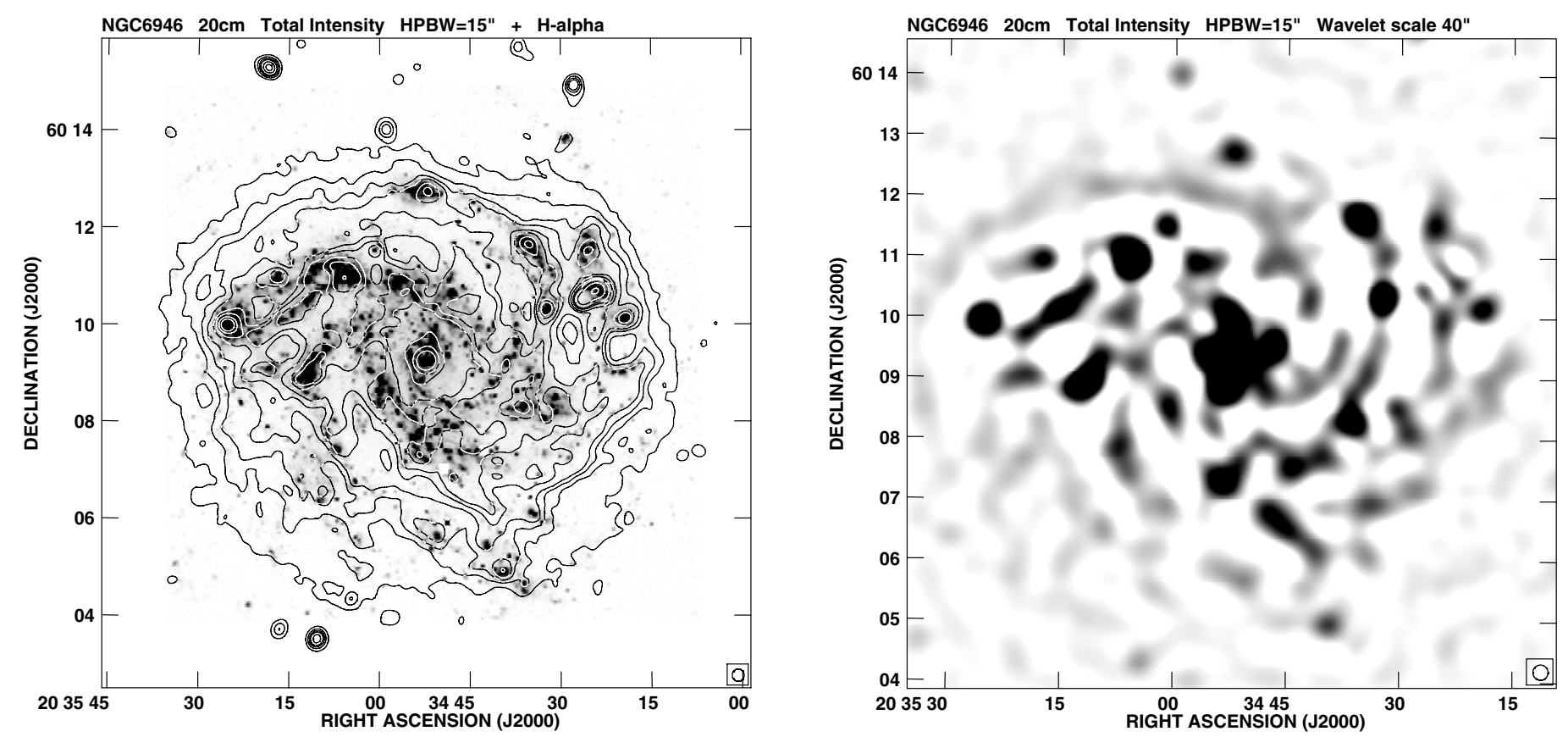

Fig. 2. Left: total intensity contours of NGC 6946 at $\lambda 20.5 \mathrm{~cm}$ at $15^{\prime \prime}$ resolution, combined from VLA and Effelsberg data. The contour intervals are $1,2,3,4,6,8,12,16,32,64,128 \times 200 \mu \mathrm{Jy} /$ beam area . The background greyscale image shows the $\mathrm{H} \alpha$ emission (Ferguson et al. 1998). Right: filtered version of the $\lambda 20.5 \mathrm{~cm}$ image by subtracting the three brightest sources in the fields and transformation with an isotropic "Mexican hat" wavelet function with a half-power width of $40^{\prime \prime}$ (see Frick et al. 2001).

NGC 6946 is surrounded by an envelope of weak radio emission which is best visible in the most sensitive $\lambda 20.5 \mathrm{~cm}$ map (Fig. 2 left) and is mostly of nonthermal synchrotron origin, as evidenced by its steep spectral index (Fig. 3). Walsh et al. (2002) found that the exponential scale length of the variation of the nonthermal emission at $\lambda 20.5 \mathrm{~cm}$ with galactocentric radius is $\simeq 4 \mathrm{kpc}$, about twice that of the $\mathrm{H} \alpha$, CO and IR emission components. In case of energy equipartition between magnetic fields and total cosmic rays (and constant ratio of cosmicray protons to electrons), the scale lengths of the cosmic-ray electrons and of the total magnetic energy density are $\simeq 8 \mathrm{kpc}$, $\left(3-\alpha_{\mathrm{n}}\right) / 2 \simeq 2$ times longer than that of the nonthermal emission (where $\alpha_{\mathrm{n}} \simeq-1$ is the synchrotron spectral index, see below), and the scale length of the total magnetic field strength is $\simeq 16 \mathrm{kpc}, 3-\alpha_{\mathrm{n}} \simeq 4$ times longer than that of the nonthermal emission (see Fig. 5). If the electrons are mostly generated in the inner disk where star formation is strongest and lose energy when diffusing outwards, their scale length is smaller than that obtained from the equipartition estimate while that of the magnetic field is even larger than $16 \mathrm{kpc}$.

In the logarithmic representation (Fig. 1 right) a weak, regular feature appears between the two northern spiral arms. The wavelet-filtered $\lambda 20.5 \mathrm{~cm}$ image of scales of around $40^{\prime \prime}$ $(\simeq 1 \mathrm{kpc}$ ) shows this feature much clearer. It is the northern magnetic arm which is highly polarized and hence much more prominent in polarized intensity (Sect. 3.4).

An almost circular hole with very low radio emission is observed at RA, $\operatorname{Dec}(2000)=20^{\mathrm{h}} 34^{\mathrm{m}} 20^{\mathrm{s}}, 60^{\circ} 09^{\prime} 40^{\prime \prime}$ (Figs. 1 and 2). Its diameter is about $40^{\prime \prime}(1 \mathrm{kpc})$. As cosmic-ray electrons can easily traverse $1 \mathrm{kpc}$ distance, the lack of synchrotron emission must be the result of a locally weak magnetic field. A highly regular field perpendicular to the disk, almost along the line of sight and hence without observable radio emission, is another explanation, though less probable. The hole is also very prominent in $\mathrm{H}_{\mathrm{I}}$ (Braun et al. 2007) and probably represents

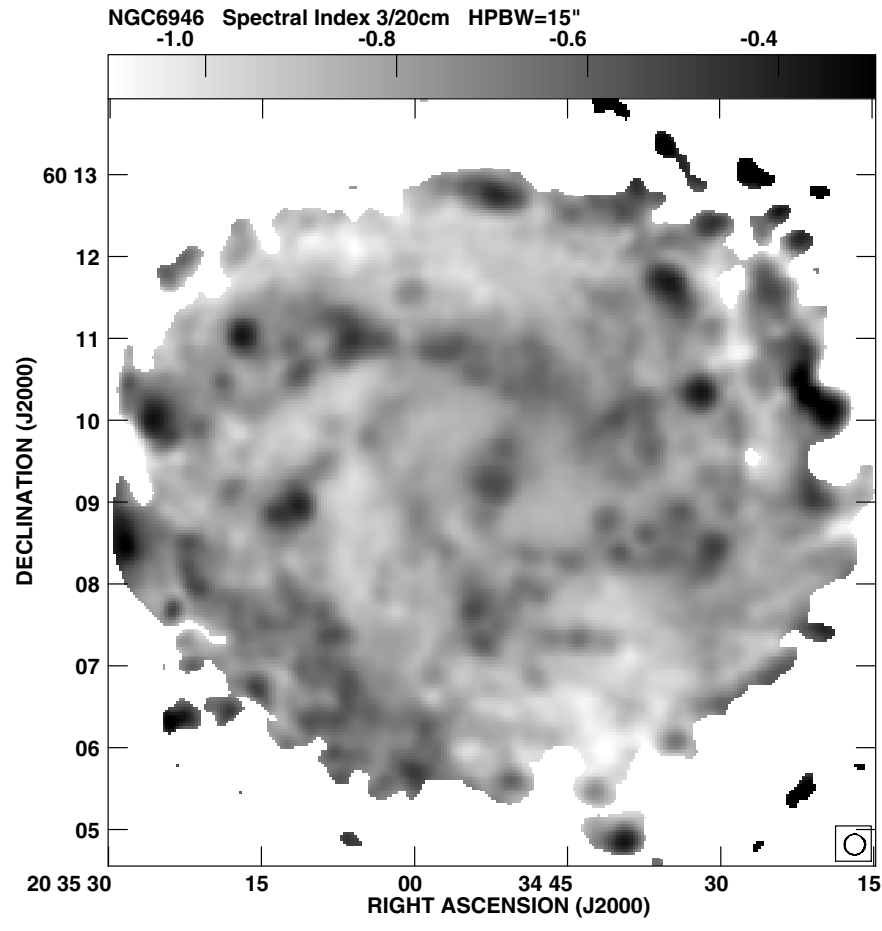

Fig. 3. Spectral index distribution between $\lambda 20 \mathrm{~cm}$ and $\lambda 3.5 \mathrm{~cm}$ at $15^{\prime \prime}$ resolution, determined for points where the intensities at both wavelengths exceed $10 \times$ the rms noise, so that the maximum error is \pm 0.08 .

a superbubble driven by multiple supernova explosions which swept away the gas and magnetic fields. Hi holes are a common phenomenon in galaxies like NGC 6946 (Kamphuis \& Sancisi 1993 ), but the association with a hole in radio continuum is rare. 


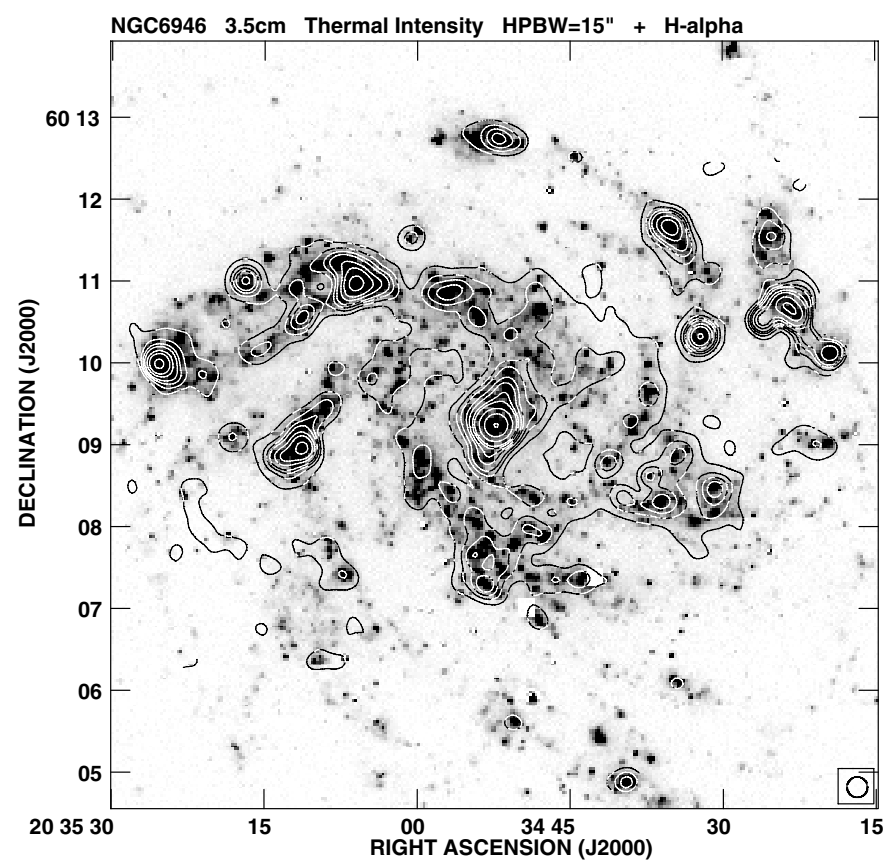

Fig. 4. Contours of thermal intensity of NGC 6946 at $\lambda 3.5 \mathrm{~cm}$ at $15^{\prime \prime}$ resolution. The contour intervals are $1,2,3,4,6,8,12,16,32,64,128 \times$ $150 \mu \mathrm{Jy} /$ beam area. The background greyscale image shows the $\mathrm{H} \alpha$ emission (Ferguson et al. 1998).

\subsection{Thermal and nonthermal emission}

The maps of intensities $I_{v}$ at $\lambda 20 \mathrm{~cm}$ and $\lambda 3.5 \mathrm{~cm}$ at $15^{\prime \prime}$ resolution were used to determine the radio spectral index $\alpha$ (Fig. 3). The spectrum is generally flatter in the spiral arms than outside the arms, indicating a larger fraction of thermal emission in the arms.

The thermal and nonthermal components were separated in the classical way by assuming a constant spectral index of -0.1 for the thermal and -1.0 for the diffuse nonthermal emission, for which the spectral index of the outer disk was taken. The resulting map of thermal emission agrees well with the distribution of $\mathrm{H} \alpha$ emission (Fig. 4). Frick et al. (2001) showed that the wavelet cross-correlation between radio and optical thermal emission is very high for scales larger than about $40^{\prime \prime}(\simeq 1 \mathrm{kpc})$; the weaker correlation at smaller scales is probably due to enhanced extinction in large, dense star-forming regions.

The assumption of a constant nonthermal spectral index $\alpha_{\mathrm{n}}=-1.0$ is clearly too simplistic. Firstly, the regions of high star-formation rate in the spiral arms contain a large fraction of young cosmic-ray electrons released from supernova remnants which have a flat spectrum of $\alpha_{\mathrm{n}} \simeq-0.6$ (Beck \& Krause 2005). Away from the spiral arms electrons lose energy by synchrotron, inverse Compton and escape losses, all of which are energy dependent and hence steepen the spectrum. Secondly, the spiral arms contain a contribution of direct synchrotron emission from supernova remnants which have spectral indices $\alpha_{\mathrm{n}}$ between 0 and -0.9 (Green 2001). Both effects lead to an overestimate of the thermal emission in the spiral arms and hence an underestimate of the nonthermal emission. More reliable estimates of the thermal radio emission require extinction-corrected $\mathrm{H} \alpha$ maps (Tabatabaei et al. 2007).

\subsection{Magnetic field strengths and energy densities}

The strengths of the total magnetic field $B_{t}$ and its regular ${ }^{3}$ component $B_{\text {reg }}$ in the sky plane can be derived from the total synchrotron intensity and its degree of linear polarization (see Sect. 3.4), assuming equipartition between the energy densities of the magnetic field and the total cosmic rays, a value for the ratio $R$ between the number densities of cosmic-ray protons and electrons, the pathlength $L$ through the synchrotron-emitting medium and the synchrotron spectral index $\alpha_{\mathrm{n}}$ (Beck \& Krause 2005). With $R=100, L=1 \mathrm{kpc}$ and $\alpha_{\mathrm{n}}=-1$, the average total field strength varies from $25 \mu \mathrm{G}$ in the inner bar to $15 \mu \mathrm{G}$ in a ring at $5 \mathrm{kpc}\left(\simeq 3^{\prime}\right)$ radius of $0.5 \mathrm{kpc}$ width, the average ordered field from $8 \mu \mathrm{G}$ near the centre to $6 \mu \mathrm{G}$ in the same ring at $5 \mathrm{kpc}$ radius. Typical values of the total field strength in the main optical spiral arms are $18-20 \mu \mathrm{G}$, those of the ordered field $\leq 5 \mu \mathrm{G}$. The strengths of the ordered, mostly regular field in the two main magnetic arms (which are strongest at around $5 \mathrm{kpc}$ radius) is typically $8-10 \mu \mathrm{G}$.

The faintest total intensity of $\simeq 1 \mathrm{mJy}$ per $147^{\prime \prime}$ beam detected in the outer galaxy with the Effelsberg telescope at $\lambda 6.2 \mathrm{~cm}$ is near the confusion limit of unresolved background sources of $0.5 \mathrm{mJy} /$ beam area with this telescope (Reich, priv. comm.) and gives a detection limit for total (equipartition) fields of about $6 \mu \mathrm{G}$. The Effelsberg total intensity limit corresponds to $\simeq 10 \mu \mathrm{Jy}$ per $15^{\prime \prime}$ beam, which is below the rms noise of the VLA maps in this paper. The faintest polarized intensity (Fig. 8) of $\simeq 200 \mu \mathrm{Jy}$ per $147^{\prime \prime}$ beam (corresponding to $\simeq 2 \mu \mathrm{Jy}$ per $15^{\prime \prime}$ beam) gives a detection limit for ordered fields of about $3 \mu \mathrm{G}$. Detection of weaker total or ordered fields in galaxies would need unrealistically deep integrations. Detection of weaker regular fields by their intrinsic Faraday rotation of polarized emission from background sources is possible but also requires deep integrations to obtain a sufficiently large number of background sources. Hence, the data of NGC 6946 presented in this paper demonstrate the limits of present-day telescopes.

The relative importance of various competing forces in the interstellar medium can be estimated by comparing the corresponding energy densities or pressures. In the local Milky Way, the average pressures of turbulent gas motions, cosmic rays and magnetic fields are similar $\left(\simeq 1 \times 10^{-12} \mathrm{erg} \mathrm{cm}^{-3}\right)$, while the thermal pressure of the warm gas is smaller by a factor of 3-5 (Boulares \& Cox 1990; Cox 2005). In numerical MHD simulations, much of the gas falls off the equipartition lines between local thermal and magnetic pressures as well as between local thermal and kinetic pressures, and even the average values do not agree (Avillez \& Breitschwerdt 2005; Mac Low et al. 2005). On the other hand, these simulations do not include dynamo action which may help to balance magnetic and kinetic energies. The new data available for NGC 6946 allow a study of average pressures as a function of galacto-centric radius (Fig. 5), results which are hard to achieve for the Milky Way.

The energy density of the total equipartition magnetic field $\left(B_{\mathrm{t}}^{2} / 8 \pi\right)$ in the disk, identical to that of the total cosmic rays,

\footnotetext{
${ }^{3}$ Note that $B$-vectors of linearly polarized emission can trace either regular magnetic fields (i.e. preserving their direction within the telescope beam, also called coherent fields) or anisotropic fields (i.e. with multiple field reversals within the beam, also called incoherent fields). To distinguish between these two components, additional Faraday rotation data is needed. The fields observed in polarization are called "ordered" throughout this paper. If Faraday rotation data are available, "regular" or "anisotropic" are used. - Also note that the equipartition strengths of the ordered field are always lower limits due to the limited resolution (beam depolarization) and Faraday depolarization effects.
} 


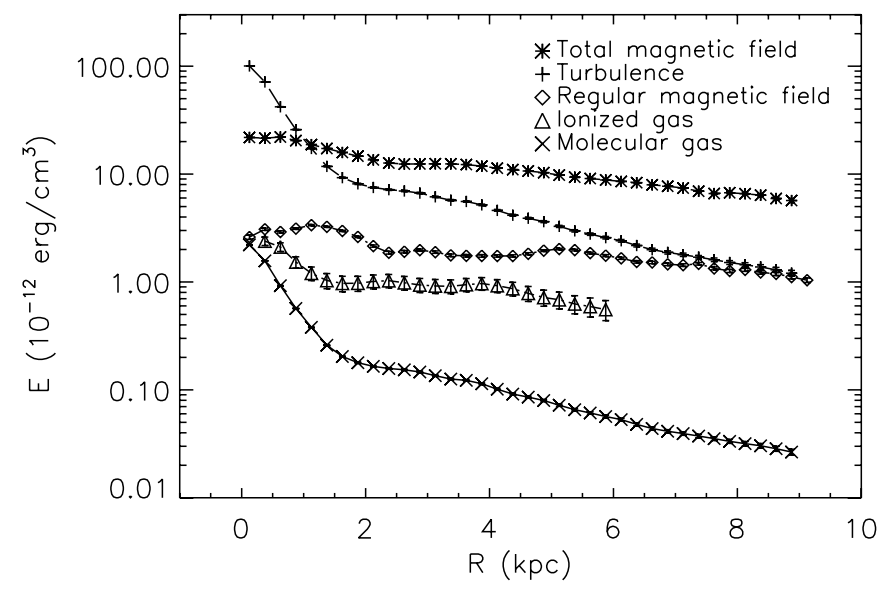

Fig. 5. Energy densities and their radial variations in the spiral galaxy NGC 6946.

was derived from the map of nonthermal synchrotron intensity, assuming a constant pathlength through the emitting medium of $1 \mathrm{kpc}$. The energy density of the ordered magnetic field was derived from the map of polarized intensity. The thermal energy density $\left(\frac{3}{2}\left\langle n_{\mathrm{e}}\right\rangle k T\right)$ of the warm ionized gas $\left(T \simeq 10^{4} \mathrm{~K}\right)$ was calculated from the map of thermal radio emission (giving the emission measure $E M=L\left\langle n_{\mathrm{e}}^{2}\right\rangle$ which is dominated by $\mathrm{H}_{\text {II }}$ regions, see Fig. 4), using a pathlength $L$ of $1 \mathrm{kpc}$, a constant volume filling factor $f$ of $5 \%$ (Ehle \& Beck 1993). The average number density $\left\langle n_{\mathrm{e}}\right\rangle=\sqrt{f\left\langle n_{\mathrm{e}}^{2}\right\rangle}$ of the $\mathrm{H}$ II regions decreases from about $0.5 \mathrm{~cm}^{-3}$ at $1 \mathrm{kpc}$ radius to about $0.3 \mathrm{~cm}^{-3}$ at $5 \mathrm{kpc}$ radius. The average number density $\langle n\rangle$ of the total neutral gas (molecular + atomic) was determined from the CO map of Walsh et al. (2002) and the H I map of Kamphuis \& Sancisi (1993), assuming for simplicity a constant scale height of the disk of $100 \mathrm{pc}$. $\langle n\rangle$ decreases from about $50 \mathrm{~cm}^{-3}$ at $1 \mathrm{kpc}$ radius to about $7 \mathrm{~cm}^{-3}$ at $5 \mathrm{kpc}$ radius. The molecular gas traced by the $\mathrm{CO}$ emission dominates until $5 \mathrm{kpc}$ radius. Assuming a constant temperature of $T=50 \mathrm{~K}$, the energy density of the molecular gas was computed and is shown in Fig. 5. The contributions of the cold and warm atomic gas cannot be determined with the existing low-resolution data, so that the thermal energy density of the total neutral gas is unknown in NGC 6946. To compute the kinetic energy density $\left(\frac{1}{2} \rho v^{2}\right)$ of the turbulent motion of the total neutral gas, its turbulent velocity was assumed to be $v_{\text {turb }}=7 \mathrm{~km} \mathrm{~s}^{-1}$, the typical value derived for the neutral gas in our Galaxy (Boulares \& Cox 1990; Kalberla \& Kerp 1998) and from ISM models (Fletcher \& Shukurov 2001).

The energy densities of all components in NGC 6946 are about one order of magnitude larger than in the Milky Way (Cox 2005). The energy density of the ionized gas $E_{\text {th }}$ in NGC 6946 (Fig. 5) is about one order of magnitude smaller than that of the magnetic field $E_{\text {magn }}$. This result is similar to that derived for the Milky Way (Cox 2005). The ISM seems to be a low$\beta$ plasma $\left(\beta=E_{\mathrm{th}} / E_{\text {magn }}\right)$. 3D magnetohydrodynamical models for the ISM predicted a large variation of local $\beta$ values, with the average value steeply increasing with temperature (Mac Low et al. 2005; Avillez \& Breitschwerdt 2005).

The thermal energy density of the ionized gas $E_{\text {th }}$ in Fig. 5 and hence $\beta$ may be underestimated if there is a significant contribution from hot gas, or if the diffuse warm ionized gas dominates over the $\mathrm{H}$ II regions, so that the effective filling factor $f$ of the warm ionized gas would be larger than $5 \%\left(\left\langle n_{\mathrm{e}}\right\rangle \propto \sqrt{I_{\text {th }} f}\right)$. However, the values of $\left\langle n_{\mathrm{e}}\right\rangle$ for the H II regions in NGC 6946 (see above) are more than one order of magnitude larger than for the warm diffuse ionized gas (Sect. 3.7) and about two orders of magnitude larger than typical values for the hot gas (Fletcher $\&$ Shukurov 2001). Finally, $E_{\text {th }}$ could be twice larger if the contribution of protons is also considered. In summary, no major uncertainties in $E_{\text {th }}$ are expected.

The large energy density of the turbulent motions compared to the thermal energy density in Fig. 5 means that turbulence in the diffuse ISM is supersonic, which is consistent with 3D MHD models for the ISM (Avillez \& Breitschwerdt 2005). While supersonic turbulence in molecular gas clouds of the Milky Way is observed and helps to support the clouds (Mac Low \& Klessen 2004), supersonic turbulence in the diffuse ISM would lead to widespread shocks and hence is hard to maintain. The results presented here are puzzling and need further investigations in other galaxies.

In the inner parts of NGC 6946 the energy densities of the total magnetic field and turbulent gas motions are similar, while the field dominates in the outer parts. The reason is the large radial scale length of the total magnetic energy $(7.0 \pm 0.1 \mathrm{kpc}$ for radii larger than $3 \mathrm{kpc}$ ) compared to the scale length of about $3 \mathrm{kpc}$ of the neutral gas density (Walsh et al. 2002). This is in apparent conflict with the generation of interstellar magnetic fields from turbulent gas motions. Before considering physical explanations, like radial diffusion of the magnetic field (Priklonsky et al. 2000), field configurations in a wind-driven halo (Breitschwerdt et al. 2002), or a supra-equipartition turbulent dynamo (Belyanin et al. 1993), possible bias effects of the values shown in Fig. 5 have to be discussed.

The discrepancy between total magnetic and turbulent energy densities may indicate that the turbulent velocity of the neutral gas in NGC 6946 is underestimated. $6 \mathrm{~km} \mathrm{~s}^{-1}$ and $9 \mathrm{~km} \mathrm{~s}^{-1}$ of the cold and warm neutral gas components, respectively, are needed for the vertical hydrostatic equilibrium, while the total turbulent velocity is $\sqrt{3}$ times higher (Fletcher \& Shukurov 2001). Another increase could be caused by the high starformation rate in NGC 6946. As a result, the magnetic and turbulent energy densities could become similar until several kpc radius. In the outer parts of the galaxy, turbulence generated by the magneto-rotational instability (MRI) (Sellwood \& Balbus 1999; Dziourkevitch et al. 2004) could be stronger than turbulence excited by star formation and increase the turbulent energy density and scale length.

The discrepancy between total magnetic and turbulence energy densities for radii larger than about $2 \mathrm{kpc}$ may also indicate that the magnetic field strength or the scale length of magnetic energy density are overestimated. If energy equipartition between magnetic fields and cosmic rays does not hold, the estimates of field strength based on this assumption would be invalid. If, e.g., the density of cosmic-ray electrons is constant in the galaxy, the scale length of the magnetic energy density would decrease to $3.5 \mathrm{kpc}$, similar to the scale length of the energy density of turbulence. However, the sources of cosmic rays are believed to be related to star-forming regions, which are concentrated in the inner galaxy, and the propagation of cosmicray electrons is limited by synchrotron losses, so that a constant density of cosmic-ray electrons can hardly be maintained. $\gamma$-ray data from the Milky Way indeed indicate a radial decrease of the cosmic ray distribution (Strong \& Mattox 1996). The aging of cosmic-ray electrons towards the outer galaxy leads to a steeper decrease and hence to an increasing proton-to-electron ratio $R$ and to an underestimate of the magnetic energy as shown in Fig. 5. This would enhance the discrepancy with the turbulent energy. 
Fluctuations of the total field strength across the telescope beam or along the line of sight are a possible source of uncertainty of the equipartition estimate because the observed synchrotron emission traces $\left(\left\langle B_{\mathrm{t}}^{4}\right\rangle\right)^{1 / 4}$ (Beck \& Krause 2005, for a synchrotron spectral index $\left.\alpha_{\mathrm{n}}=-1\right)$ and hence is biased towards regions with strong fields. With fluctuations in strength of amplitude $a$ (where $a=\delta B_{\mathrm{t}} /\left\langle B_{\mathrm{t}}\right\rangle$ ) the total equipartition strength is too large by a factor of $\left(1+6 a^{2}+a^{4}\right)^{1 / 4}$. Note that $\delta B_{\mathrm{t}}$ is smaller than the random field $B_{\mathrm{r}}$ as derived from Faraday depolarization (Sect. 3.9) because fluctuations of the field direction do not contribute to $\delta B_{\mathrm{t}}$. For the same reason, the degree of field regularity (Fig. 10) also cannot be used to estimate $a$. Regarding $a \simeq 1$ as the upper limit, the maximum overestimate is 1.7 times for the total field strength and 2.8 for the total magnetic energy. In summary, there may be room to argue for an overestimate of the total magnetic energy density in Fig. 5 by a factor of about 2-3.

The energy density of the ordered field in NGC 6946 is 5-8 times smaller than that of the total field, while the scale length is similar, $8.2 \pm 0.8 \mathrm{kpc}$ for radii larger than $6 \mathrm{kpc}$. Ordered fields are detected in NGC 6946 up to about $15 \mathrm{kpc}$ radius (Fig. 8) and regular fields up to $12 \mathrm{kpc}$ radius (Sect. 3.7), but these may extend much further, to at least $20 \mathrm{kpc}(12.5)$ radius until which $\mathrm{H}_{\mathrm{I}}$ emission is detected (Boomsma et al. 2005; Braun et al. 2007, Fig. 7). A deep survey of Faraday rotation of polarized background sources is needed to search for magnetic fields at large radii.

If the slow decrease of the magnetic field energy density in Fig. 5 continues to even larger radii, it may affect the rotation curve in the outer galaxy, as proposed by Battaner \& Florido (2000). According to Battaner \& Florido (2007) the flat radial profile of the magnetic energy in NGC 6946 causes a net inward force.

\subsection{Polarized intensity}

Figures 6-11 show the distributions of the polarized emission at the five observation frequencies. There is very little similarity to the maps of total emission. The polarized emission in the optical spiral arms is low; it is anticorrelated with the $\mathrm{H} \alpha$ emission (Frick et al. 2001). The fractional polarization in the inner optical arms of $1-5 \%$ at $\lambda \lambda 3,6 \mathrm{~cm}$ wavelengths is low due to wavelength-independent depolarization by unresolved field structures, either fields tangled by star-formation activity or by turbulent fields. In the outer arms the fractional polarization increases to $5-10 \%$. At $\lambda \lambda 18,20 \mathrm{~cm}$ the fractional polarization in the arms is smaller than $2 \%$.

Note that the orientations of the observed $E$-vectors at $\lambda 18.0 \mathrm{~cm}$ (Fig. 6 bottom left) and $\lambda 20.5 \mathrm{~cm}$ are by chance similar to those of the magnetic field because Faraday rotation at these wavelengths originates mainly in the Galactic foreground which has $R M_{\mathrm{fg}} \simeq 40 \mathrm{rad} \mathrm{m}^{-2}$, giving $74^{\circ}$ at $\lambda 18.0 \mathrm{~cm}$ and $96^{\circ}$ at $\lambda 20.5 \mathrm{~cm}$ (see Sect. 3.7).

The Faraday-corrected magnetic field vectors (Fig. 6 right) appear to be less ordered than the observed $B$-vectors (Fig. 6 left). This is due to the relatively large errors in Faraday rotation which had to be determined from data over a small frequency range, leading to errors in the field orientation of up to $15^{\circ}$ between $\lambda 3 \mathrm{~cm}$ and $\lambda 6 \mathrm{~cm}$ and up to $45^{\circ}$ between $\lambda 18 \mathrm{~cm}$ and $\lambda 20 \mathrm{~cm}$ at the cutoff limit of 4 times the rms noise (Sect. 3.7).

The most striking phenomena in Fig. 6 are the two bright magnetic arms between the optical arms, known already from the previous $\lambda 6.2 \mathrm{~cm}$ observations (Beck \& Hoernes 1996). The magnetic arms are visible at all wavelengths, except in the single-dish Effelsberg maps (Figs. 8 and 9) where the beam is too large to resolve them.

The fractional polarization $p$ observed at $\lambda 3 \mathrm{~cm}$ and $\lambda 6 \mathrm{~cm}$ is $30-45 \%$ in the northern magnetic arm and $20-30 \%$ in the southern magnetic arm. These are among the highest degrees of polarization observed in any spiral galaxy so far, similarly high as in the emission ring of M 31 (Berkhuijsen et al. 2003). The fractional polarization of the nonthermal emission $p_{\mathrm{n}}$ is obtained by subtraction of the unpolarized thermal contribution. As the thermal emission is small in the interarm regions (Fig. 4) $p_{\mathrm{n}}$ is only marginally higher than $p$ in the magnetic arms. Subtraction of the smooth, mostly unpolarized background from the total intensity increases $p$ in the northern arm to about $75 \%$ at $\lambda 3 \mathrm{~cm}$ and $\lambda 6 \mathrm{~cm}$, which is the maximum possible degree of linear polarization $p_{0}$ of $p_{0}=\left(3-3 \alpha_{\mathrm{n}}\right) /\left(5-3 \alpha_{\mathrm{n}}\right)$ (where $\alpha_{\mathrm{n}}$ is the nonthermal spectral index). The magnetic field in the northern magnetic arm seems to be totally aligned.

The bright magnetic arms are located between the optical and gas spiral arms, but they are not free of gas. The H I emission is quite strong in the interarm regions of the inner galaxy, without concentration to the magnetic arms (Fig. 7). Weak interarm emission is seen in the maps of $7 \mu \mathrm{m}$ dust emission (Fig. 1 left) and in $\mathrm{H} \alpha$ (Fig. 4). Deeper images may reveal a relation between the magnetic arms and interarm gas or dust features.

At $\lambda 18.0 \mathrm{~cm}$ and $\lambda 20.5 \mathrm{~cm}$, where the signal-to-noise ratio is highest, the bright magnetic arms observed at smaller wavelengths turn out to be the brightest features of a system of at least five magnetic arms which extend to at least $10 \mathrm{kpc}$ radius (Fig. 6 bottom and Fig. 17). The main northern magnetic arm continues towards the east, $10 \mathrm{kpc}$ from the centre. Two more magnetic arms can be identified north and north-west of the main northern magnetic arm. Both outer magnetic arms are located in regions with very low density of $\mathrm{H}_{\mathrm{I}}$ gas (Fig. 7), one at the outer edge of an $\mathrm{H}$ I arm at 3.5 $(5.5 \mathrm{kpc})$ north of the centre, the other one at the inner edge of the next arm at $6^{\prime}(9.5 \mathrm{kpc})$ north-west of the centre. South-east of the main southern magnetic arm, another magnetic arm is seen which is broad and may consist of several individual features. More magnetic arms could exist in the outer south-western quadrant, but strong Faraday depolarization prevents their detection at $\lambda 18.0 \mathrm{~cm}$ and $\lambda 20.5 \mathrm{~cm}$. The two bright magnetic arms can be interpreted as a superposition of two azimuthal dynamo modes ( $m=0$ and $m=2$ ), but five magnetic arms or more need higher modes with $m \geq 3$ (see discussion in Sect. 4.1).

The maps of polarized intensities at short wavelengths (Fig. 6 top) and those at longer wavelengths (Fig. 6 bottom) differ in the south-western quadrant where Faraday depolarization $D P$ is strong at $\lambda \lambda 18.0,20.5 \mathrm{~cm}$, as already known from previous observations at lower resolution (Beck 1991). The origin of this DP asymmetry is discussed in Sect. 4.2. The Effelsberg $\lambda 6.2 \mathrm{~cm}$ and $\lambda 11.2 \mathrm{~cm}$ polarization maps (Figs. 8 and 9) do not show this hole. This indicates that Faraday depolarization is not yet strong at $\lambda 11.2 \mathrm{~cm}$.

The orientations of the field vectors (Fig. 6 right) follow well the orientation of the spiral structure seen in polarized intensity. A similar result has been found for M 51 where the large-scale spiral shock front is able to align the magnetic field (Patrikeev et al. 2006). However, spiral shocks cannot explain the field alignment along the magnetic arms of NGC 6946 (see Sect. 4.1).

\subsection{Field regularity}

$p_{\mathrm{n}}$ is a measure of the ratio $q$ of the field strength of the regular field in the sky plane and the random field, the degree of 

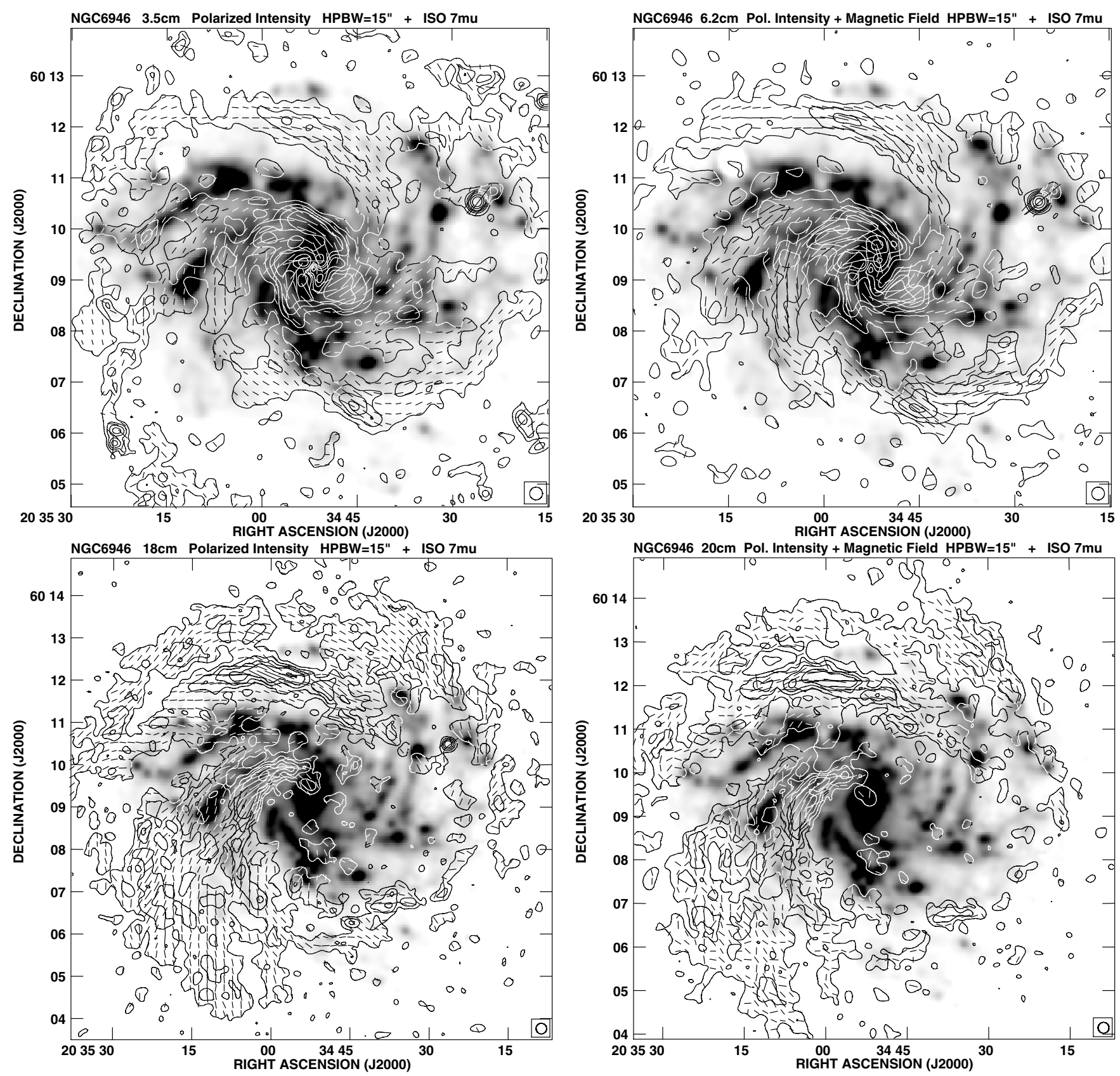

Fig. 6. Top left: Polarized intensity contours and observed $B$-vectors $\left(E+90^{\circ}\right)$ of NGC 6946 at $\lambda 3.5 \mathrm{~cm}$ at $15^{\prime \prime}$ resolution, combined from VLA and Effelsberg data. The contour intervals are 1,2,3,4,6,8 $\times 40 \mu \mathrm{Jy} /$ beam area. The vector length is proportional to polarized intensity, $12^{\prime \prime}$ length corresponds to $100 \mu \mathrm{Jy} /$ beam area. Faraday rotation is low $\left(\leq 10^{\circ}\right)$ at this wavelength. The background greyscale image shows the $7 \mu \mathrm{m}$ IR emission measured with the ISO satellite with about 6" resolution (Dale et al. 2000). Top right: Polarized intensity contours and $B$-vectors at $\lambda 6.2 \mathrm{~cm}$, corrected for Faraday rotation (see Fig. 12 left) at 15" resolution, combined from VLA and Effelsberg data. The contour intervals are $1,2,3,4,6,8 \times 60 \mu \mathrm{Jy} /$ beam area. The vector length is proportional to polarized intensity, $12^{\prime \prime}$ length corresponds to $100 \mu \mathrm{Jy} / \mathrm{beam}$ area . Bottom left: Polarized intensity contours and observed $E$-vectors at $\lambda 18.0 \mathrm{~cm}$, not corrected for Faraday rotation, at $15^{\prime \prime}$ resolution, combined from VLA

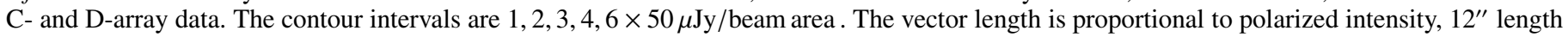
corresponds to $100 \mu \mathrm{Jy} /$ beam area. Bottom right: Polarized intensity contours and $B$-vectors at $\lambda 20.5 \mathrm{~cm}$, corrected for Faraday rotation (see Fig. 12 right), at $15^{\prime \prime}$ resolution, combined from VLA C- and D-array data. The contour intervals are 1, 2, 3, 4, $6 \times 50 \mu \mathrm{Jy} /$ beam area . The vector length is proportional to polarized intensity, $12^{\prime \prime}$ length corresponds to $100 \mu \mathrm{Jy} / \mathrm{beam}$ area .

regularity of the field, $q=B_{\text {reg }} / B_{\text {ran. }}$. As some fraction of the field observed in polarization can be an anisotropic turbulent field (see footnote (3)), $q$ as derived from $p_{\mathrm{n}}$ is an upper limit. For constant cosmic-ray energy density and an isotropic random field (Burn 1966 and Eq. (2) in Beck et al. 2003):

$p_{\mathrm{n}} / p_{0}=q^{2} /\left(q^{2}+\frac{2}{3}\right)$

$$
q=\left\{2\left(p_{\mathrm{n}} / p_{0}\right) /\left[3\left(1-\left(p_{\mathrm{n}} / p_{0}\right)\right)\right]\right\}^{0.5} .
$$

$p_{0}$ is the intrinsic degree of polarization; $p_{0}=\left(3+3 \alpha_{\mathrm{n}}\right) /\left(5+3 \alpha_{\mathrm{n}}\right)$, where $\alpha_{\mathrm{n}}$ is the nonthermal spectral index. In case of equipartition between the energy densities of magnetic field and cosmic 


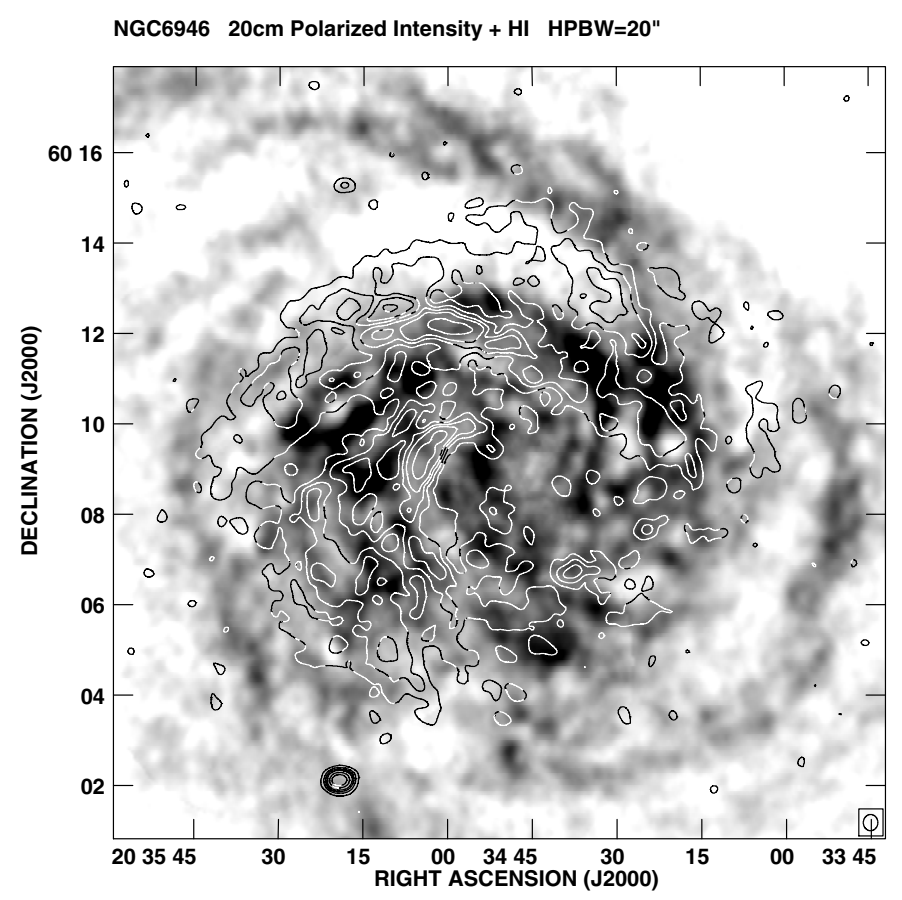

Fig. 7. Polarized intensity contours of NGC 6946 at $\lambda 20.5 \mathrm{~cm}$ at $20^{\prime \prime}$ resolution, observed with VLA. The contour intervals are 1, 2, 3, 4,6,8 $\times$ $60 \mu \mathrm{Jy} /$ beam area. The background greyscale image shows the $\mathrm{H}_{\mathrm{I}}$ emission measured with the Westerbork telescope at about the same resolution (Boomsma et al. 2005; Braun et al. 2007).

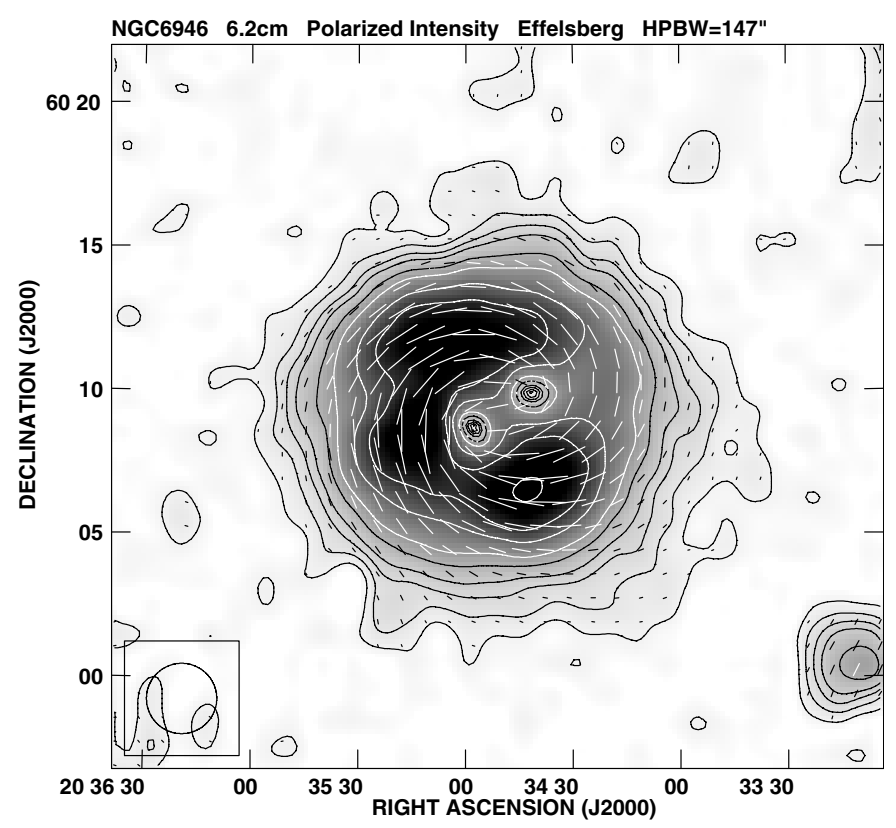

Fig. 8. Polarized intensity contours and $B$-vectors $\left(E+90^{\circ}\right)$ of NGC 6946 at $\lambda 6.2 \mathrm{~cm}$ at 2'.45 resolution, observed with the Effelsberg telescope. The contour intervals are $1,2,3,4,6,8,12,16,24 \times$ $200 \mu \mathrm{Jy} /$ beam area. The vector length is proportional to polarized intensity, $20^{\prime \prime}$ length corresponds to $1000 \mu \mathrm{Jy} /$ beam area. The vector orientations are not corrected for Faraday rotation.

rays (Eq. (21) in Beck et al. 2003, adding the missing term $q^{-2}$ ):

$$
\begin{aligned}
p_{\mathrm{n}} / p_{0} & =\left(q^{2}+\frac{7}{3}\right) /\left(q^{2}+3+\frac{10}{9} q^{-2}\right) \simeq q^{2} /\left(q^{2}+\frac{1}{2}\right) \\
q & \simeq\left\{\left(p_{\mathrm{n}} / p_{0}\right) /\left[2\left(1-\left(p_{\mathrm{n}} / p_{0}\right)\right)\right]\right\}^{0.5},
\end{aligned}
$$

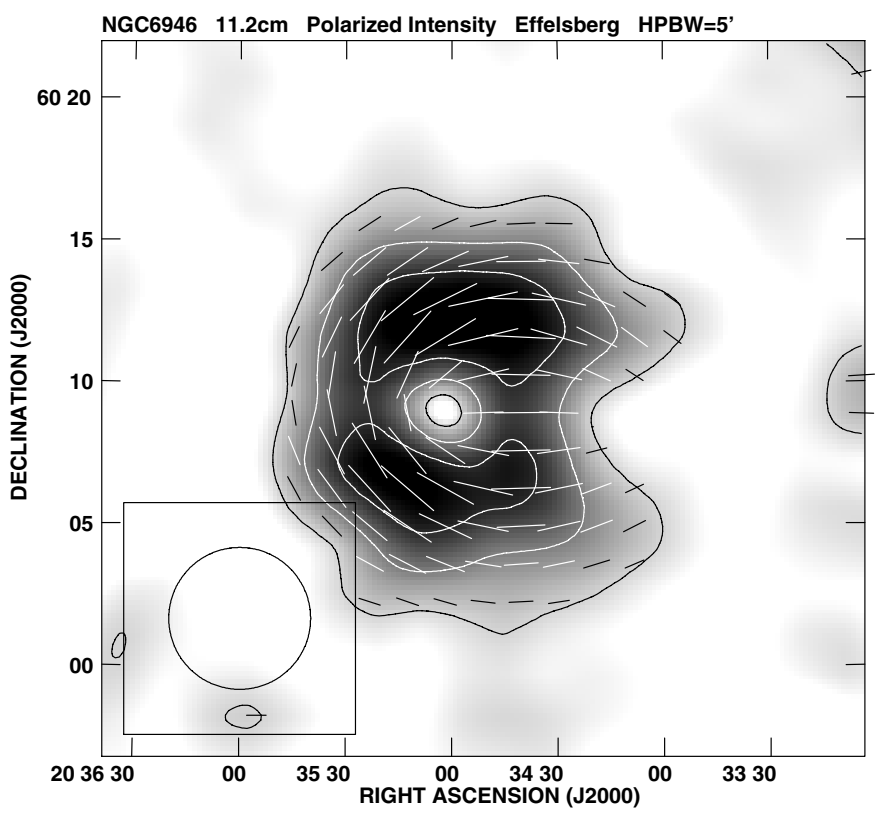

Fig. 9. Polarized intensity contours and $B$-vectors $\left(E+90^{\circ}\right)$ of NGC 6946 at $\lambda 11.2 \mathrm{~cm}$ at $5^{\prime}$ resolution, observed with the Effelsberg telescope. The contour intervals are 1,2,3×2000 $\mu \mathrm{Jy} /$ beam area. The vector length is proportional to polarized intensity, $1^{\prime}$ length corresponds to $3000 \mu \mathrm{Jy} /$ beam area. The vector orientations are not corrected for Faraday rotation.

where the approximation is accurate to better than $5 \%$ for $q<$ $0.1,2-5 \%$ for $0.1 \leq q \leq 1$ and better than $2 \%$ for $q>1$. For the same value of $p_{\mathrm{n}}$, the field regularity $q$ in case of equipartition is smaller by 0.87 times compared to the value for constant cosmic-ray density. For small values of $q$ and in case of equipartition, $p_{\mathrm{n}}$ is larger by 1.33 times compared to constant cosmicray density.

Figure 10 shows the degree of regularity $q$ of the field, derived from the fractional polarization of the nonthermal emission at $\lambda 6.2 \mathrm{~cm}$, using Eq. (4) for the case of equipartition. $q$ is generally below 1 over most of the galaxy, but increases to about 1 in the outer parts of the two main magnetic arms. In the local Milky Way, starlight polarization and radio synchrotron data yield an average value of $q \simeq 0.6$ (see Beck 2001 for a review).

\subsection{Field compression in the inner spiral arms}

At the highest available resolution at $\lambda 3.5 \mathrm{~cm}$, the spiral pattern of the magnetic field continues well into the centre of NGC 6946 (Fig. 11). The polarized intensity is very diffuse and fills almost the whole area, but avoiding the optical spiral arms. High polarized intensities are found at the inner edge of the inner spiral arm seen in Fig. 11 north of the centre, but only along a small part of the southern inner optical arm. The $B$-vectors are aligned along the northern optical arm, giving evidence that the field is compressed or sheared, but Faraday rotation is not enhanced in this region (see Fig. 12 left). This means that the field along the optical spiral arm is anisotropic turbulent, with frequent (unresolved) reversals, as expected from compression or shear of an isotropic turbulent field. The compressed fields in the bars of NGC 1097 and NGC 1365 (Beck et al. 2005) and in the massive inner spiral arms of M 51 (Fletcher et al. 2007) have similar properties. Polarized radio emission is an ideal tracer of field compression or shear. Polarization also nicely traces 


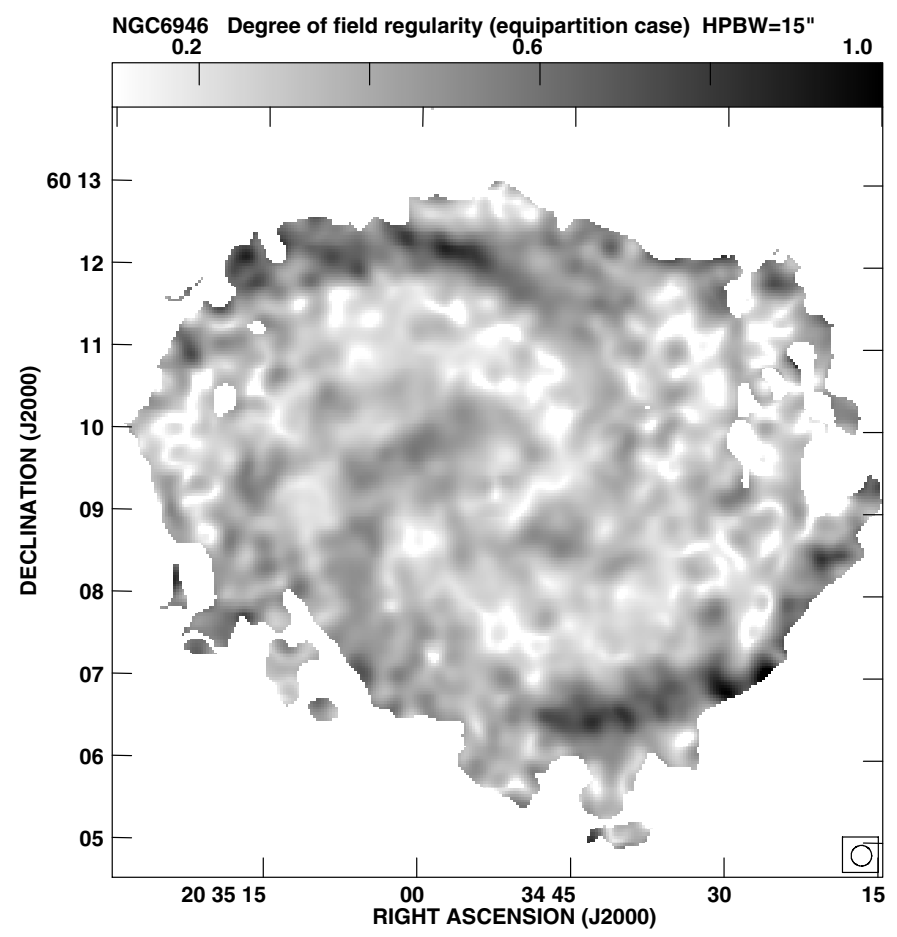

Fig. 10. Degree of field regularity $q$, the ratio of the regular field strength in the sky plane to the isotropic random field strength, derived from the fractional polarization of the nonthermal intensity of NGC 6946 at $\lambda 6.2 \mathrm{~cm}$ at $15^{\prime \prime}$ resolution. $q$ was computed only at points where the total nonthermal intensity at $\lambda 6.2 \mathrm{~cm}$ exceeds 10 times the rms noise.

interactions between galaxies or with the intergalactic medium (Vollmer et al. 2007).

The contrast in polarized intensity between the northern inner arm and the diffuse region inside of the arm is about 2 . Assuming either constant cosmic rays or energy equipartition between magnetic fields and cosmic rays, this corresponds to a mild compression of the magnetic field by $1.4 \times$ and $1.2 \times$, respectively.

The $B$-vectors in Fig. 11 are not sharply deflected in the compression region, as expected for a shock front, but turn smoothly from the upstream region (east of the northern arm) into the compression region. A similar observation in barred galaxies lead to the conclusion that the regular field is not coupled to the cold, molecular gas but to the warm, diffuse gas (Beck et al. 2005).

The hole in polarized intensity south-west of the nucleus (Fig. 11) is the result of beam depolarization, as the field is strongly twisted within the telescope beam. The offset with respect to the galaxy nucleus could be due to asymmetry of the field pattern, possibly due to shearing gas flows near the inner stellar bar which is also observed in CO lines (Schinnerer et al. 2006). The generation of strong fields with a large-scale spiral pattern is typical for late-type barred galaxies (Beck et al. 2002, 2005).

\subsection{Faraday rotation}

The polarization angles at $\lambda 3.5 \mathrm{~cm}$ and $\lambda 6.2 \mathrm{~cm}$ and those at $\lambda 18.0 \mathrm{~cm}$ and $\lambda 20.5 \mathrm{~cm}$ were combined to maps of Faraday rotation measures $R M$. Clipping below 4 times the rms noise in polarized intensity restricts the maximum $R M$ error to $\pm 69 \mathrm{rad} \mathrm{m}^{-2}$ and $\pm 19 \mathrm{rad} \mathrm{m}^{-2}$, respectively. The average value in both maps of $+40 \pm 2 \mathrm{rad} \mathrm{m}^{-2}$ has been adopted as the foreground $R M_{\mathrm{fg}}$ from our Galaxy, consistent with previous results (Beck 1991;

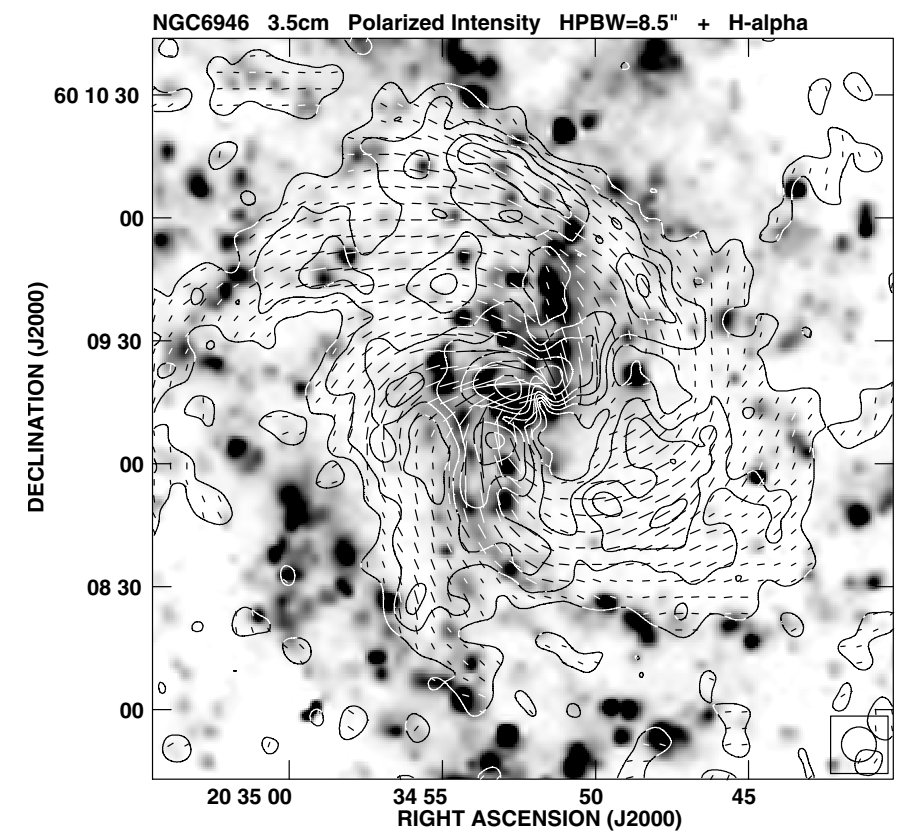

Fig. 11. Polarized intensity contours and observed $B$-vectors $\left(E+90^{\circ}\right)$ of NGC 6946 at $\lambda 3.5 \mathrm{~cm}$ at 8.5 resolution, observed with the VLA D-array. Faraday rotation is smaller than $5^{\circ}$ at this wavelength. The contour intervals are $1,2,3,4,6,8,12 \times 20 \mu \mathrm{Jy} /$ beam area. The vector length is proportional to polarized intensity, $5^{\prime \prime}$ length corresponds to $100 \mu \mathrm{Jy} /$ beam area. The background greyscale image shows the $\mathrm{H} \alpha$ emission (Ferguson et al. 1998). The position of the radio nucleus of the galaxy is RA, $\operatorname{Dec}(2000)=20^{\mathrm{h}} 34^{\mathrm{m}} 52^{\mathrm{s}} .25,+60^{\circ} 09^{\prime} 14^{\prime \prime} .5$.

Ehle \& Beck 1993), and has been subtracted to obtain Faraday rotation measures $R M_{i}$ intrinsic to NGC 6946 (Fig. 12). The ambiguity of $R M$ values between $\lambda 3.5 \mathrm{~cm}$ and $\lambda 6.2 \mathrm{~cm}$ of $n$ times $\pm 1232 \mathrm{rad} \mathrm{m}^{-2}$ due to the $\pm \pi n$ ambiguity of polarization vectors is large and can be excluded.

$R M$ errors are large due to the small wavelength difference. Noise in the maps of polarized intensity accounts for $R M$ fluctuations of $\Delta R M=\left(\sqrt{2} S\left(\lambda_{2}^{2}-\lambda_{1}^{2}\right)\right)^{-1}$ where $S$ is the signal-tonoise ratio of the polarized intensities at $\lambda_{1}$ and $\lambda_{2}$. The rms noise in $P I$ is taken to be the mean rms noise in $Q$ and $U$. $\triangle R M$ between $\lambda 3.5 \mathrm{~cm}$ and $\lambda 6.2 \mathrm{~cm}$ due to noise is expected to increase from $12 \mathrm{rad} \mathrm{m}^{-2}$ in the brightest inner parts of the northern magnetic arm $(S \simeq 23)$, to $46 \mathrm{rad} \mathrm{m}^{-2}$ over the whole northern arm $\left(S \simeq 6\right.$ ), and to $69 \mathrm{rad} \mathrm{m}^{-2}$ at the cutoff limit in Fig. 12 of $S=4$. In the brightest part of the northern magnetic arm the observed dispersion in $R M$ (Fig. $12 \mathrm{left}$ ) is $25 \mathrm{rad} \mathrm{m}^{-2}$, so that the contribution by random fields is $\simeq 10 \mathrm{rad} \mathrm{m}^{-2}$, while the dispersion of $\simeq 50 \mathrm{rad} \mathrm{m}^{-2}$ observed over the whole arm is dominated by fluctuations due to noise. A similar conclusion is valid for the fluctuations in the $R M$ map between $\lambda 18 \mathrm{~cm}$ and $\lambda 20 \mathrm{~cm}$ (Fig. 12 right).

$R M_{i}$ between $\lambda 3.5 \mathrm{~cm}$ and $\lambda 6.2 \mathrm{~cm}$ (Fig. 12 left) shows the well-known north-south asymmetry with predominantly positive values in the northern magnetic arm and negative ones in the southern magnetic arm. The large-scale variation of $R M$ with azimuthal angle in the galaxy plane is sinusoidal (Ehle \& Beck 1993). This has been interpreted as a superposition of the $m=0$ and $m=2$ dynamo modes (Rohde et al. 1999). No other largescale field reversals are observed.

The $R M$ amplitude of $\pm 100 \mathrm{rad} \mathrm{m}^{-2}$ is provided by a regular field of about $8 \mu \mathrm{G}$ strength (Sect. 3.3) and diffuse ionized gas with an electron density of $\left\langle n_{\mathrm{e}}\right\rangle \simeq 0.03 \mathrm{~cm}^{-3}$ and a pathlength of about $1 \mathrm{kpc}$. 

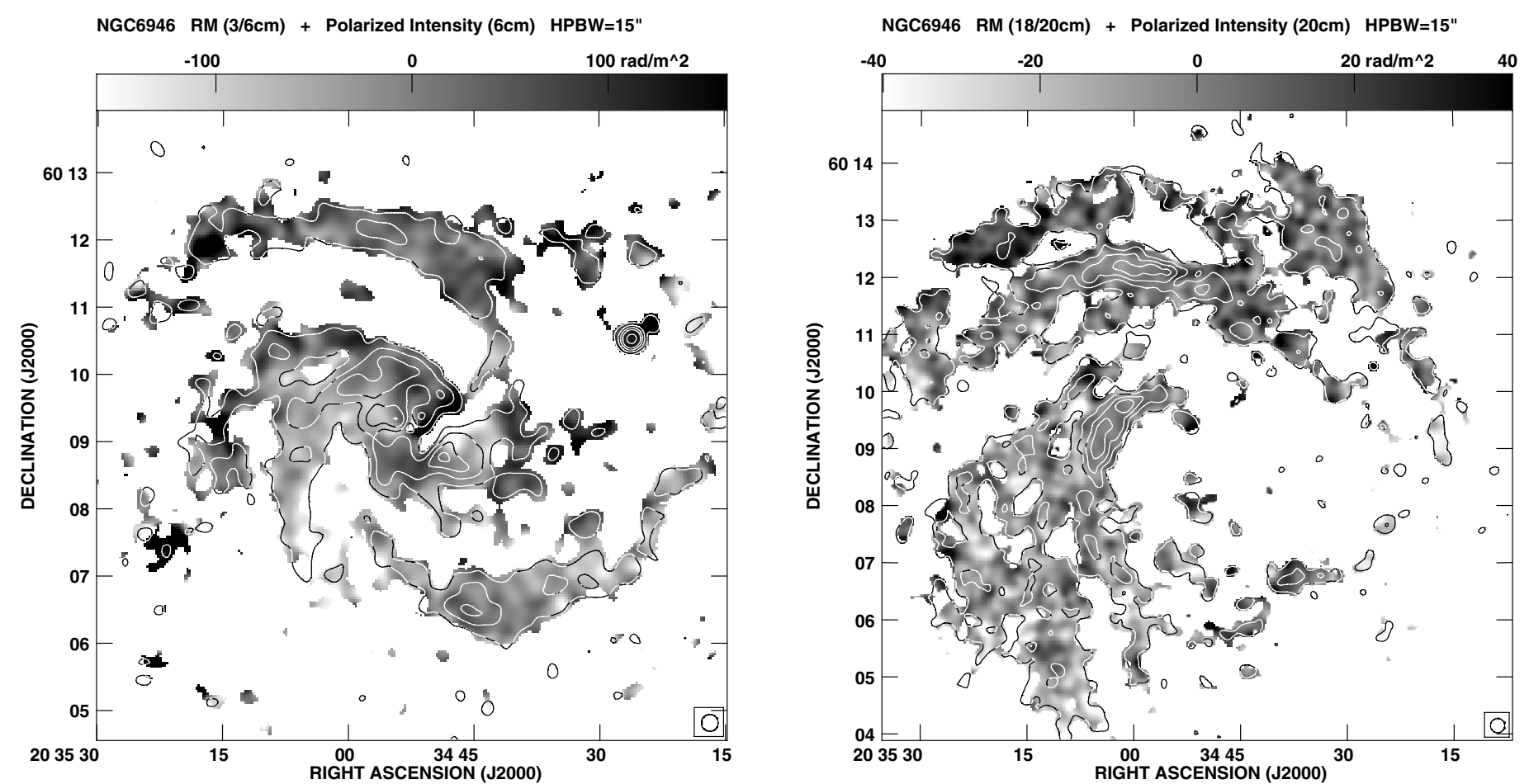

Fig. 12. Left: Intrinsic Faraday rotation measures $R M_{i}$ between $\lambda 3.5 \mathrm{~cm}$ and $\lambda 6.2 \mathrm{~cm}$ at $15^{\prime \prime}$ resolution at points where the polarized intensities at both wavelengths exceeds 4 times the rms noise (taken from that in $Q$ and $U$ ). The contours show the polarized intensity at $\lambda 6.2 \mathrm{~cm}$. The contour intervals are $1,2,3,4,6 \times 80 \mu \mathrm{Jy} /$ beam area. The foreground $R M_{\mathrm{fg}}$ from our Galaxy of $40 \mathrm{rad} \mathrm{m}^{-2}$ has been subtracted. Right: Intrinsic Faraday rotation measures $R M_{i}$ between $\lambda 18.0 \mathrm{~cm}$ and $\lambda 20.5 \mathrm{~cm}$ at $15^{\prime \prime}$ resolution at points where the polarized intensities at both wavelengths exceeds 4 times the rms noise. The contours show the polarized intensity at $\lambda 20.5 \mathrm{~cm}$. The contour intervals are $1,2,3,4 \times 60 \mu \mathrm{Jy} / \mathrm{beam}$ area. The foreground $R M_{\mathrm{fg}}$ from our Galaxy of $40 \mathrm{rad} \mathrm{m}^{-2}$ has been subtracted.

In the Milky Way, Faraday rotation data from pulsars indicate multiple large-scale field reversals (Han et al. 2006). Nothing similar has been observed in any external galaxy so far. Although Faraday rotation data in galaxies like NGC 6946 are line-of-sight integrals, large-scale field reversals would be evident from the existing data. Furthermore, the general concentration of coherent fields in interarm regions, like in many spiral galaxies, was not found in the Milky Way. Either our Galaxy is special, or the data are not yet sufficient to trace the large-scale Galactic field.

$R M_{i}$ between $\lambda 18.0 \mathrm{~cm}$ and $\lambda 20.5 \mathrm{~cm}$ (Fig. 12 right) is on average 5 times smaller than $R M_{i}$ between $\lambda 3.5 \mathrm{~cm}$ and $\lambda 6.2 \mathrm{~cm}$, which is the effect of Faraday dispersion in the disk (Sect. 3.9). In the south-west quadrant no data are available due to the weak polarized emission at $\lambda 18.0 \mathrm{~cm}$ and $\lambda 20.5 \mathrm{~cm}$. At a resolution of $72^{\prime \prime} R M_{i}$ drops to about $-50 \mathrm{rad} \mathrm{m}^{-2}$ in this region (Beck 1991).

A close pair of bright background sources (source A: RA, $\operatorname{Dec}(2000)=20^{\mathrm{h}} 34^{\mathrm{m}} 24^{\mathrm{s}} 2,+60^{\circ} 10^{\prime} 41^{\prime \prime}$, source B: $20^{\mathrm{h}} 34^{\mathrm{m}} 26^{\mathrm{s}} .1$, $\left.+60^{\circ} 10^{\prime} 32^{\prime \prime}\right)$ has fractional polarizations of $2 \%$ and $12 \%$ at both $\lambda 3.5 \mathrm{~cm}$ and $\lambda 6.2 \mathrm{~cm}$. Both sources are completely depolarized at $\lambda 20 \mathrm{~cm}$. At $\lambda 18 \mathrm{~cm}$ only source B is still visible $(p \simeq 3 \%$ ). Their $R M$ between $\lambda 3.5 \mathrm{~cm}$ and $\lambda 6.2 \mathrm{~cm}$ is $252 \pm 20 \mathrm{rad} \mathrm{m}^{-2}$ and $171 \pm 2 \mathrm{rad} \mathrm{m}^{-2}$. As polarized waves from background sources travel through the whole disk, their $R M_{0}$ (after subtraction of $R M_{\mathrm{fg}}$ ) should be about twice that of the internal emission $R M_{i}$. While the high $R M_{0}$ of source $\mathrm{A}$ is probably intrinsic, $R M_{0} \simeq$ $131 \mathrm{rad} \mathrm{m}^{-2}$ of source $\mathrm{B}$ corresponds to $R M_{i} \simeq 65 \mathrm{rad} \mathrm{m}^{-2}$ of the diffuse emission, consistent with the $R M_{i}$ of the diffuse emission of NGC 6946 observed in this region (Fig. 12 left).
Another pair of bright, polarized background sources, barely separated, is visible at the southern edge of the maps at $\lambda 18 \mathrm{~cm}$ and $\lambda 20 \mathrm{~cm}$ (Fig. 7), located at RA, Dec $(2000)=20^{\mathrm{h}} 35^{\mathrm{m}} 19 \mathrm{~s} .2$, $+60^{\circ} 02^{\prime} 08^{\prime \prime}$, at $12 \mathrm{kpc}$ distance from the centre of NGC 6946 . The total flux densities are $860 \pm 50 \mu \mathrm{Jy}$ at $\lambda 18 \mathrm{~cm}$ and $1070 \pm$ $40 \mu \mathrm{Jy}$ at $\lambda 20 \mathrm{~cm}$. The rotation measure, corrected for the average foreground of $R M_{\mathrm{fg}}=40 \pm 2 \mathrm{rad} \mathrm{m}^{-2}$, is $R M_{0}=-13 \pm$ $4 \mathrm{rad} \mathrm{m}^{-2}$ and may indicate that regular fields and ionized gas in NGC 6946 extend to at least $12 \mathrm{kpc}$ radius. However, a variation of $R M_{\mathrm{fg}}$ cannot be excluded. Ehle \& Beck (1993) determined $R M_{\mathrm{fg}}=30 \pm 4 \mathrm{rad} \mathrm{m}^{-2}$ for the radius range $9.5-14 \mathrm{kpc}$ which means no significant internal rotation measure $\left(R M_{0}=\right.$ $-3 \pm 6 \mathrm{rad} \mathrm{m}^{-2}$ ) towards the background sources. Observations of more polarized sources with higher sensitivity are required.

\subsection{Structure function of RM}

Faraday rotation measures are signatures of structures of coherent regular magnetic fields in an astrophysical plasma with a spectrum of scales which can be characterized in various ways. Wavelet spectra are an excellent tool to analyze intensity maps (Frick et al. 2001), but cannot be applied to $R M$ data. Analysis of $R M$ data of galaxy clusters with the autocorrelation function revealed power spectra of the magnetic field approximately of Kolmogorov type (Vogt \& Enßlin 2003). In our Galaxy, on scales of $0.5-5^{\circ}(\simeq 10-100 \mathrm{pc}$ at $1 \mathrm{kpc}$ distance $)$, Haverkorn et al. (2006) found slopes between 0.4 and 0.8 in interarm regions, but very flat $S F s$ in optical spiral arms. The resolution obtained in NGC 6946 allows to study only scales larger than about 400 pc.

Figure 13 shows the structure function $S F$ of the intrinsic $R M_{i}$ values in NGC 6946 at the two wavelength pairs. The 


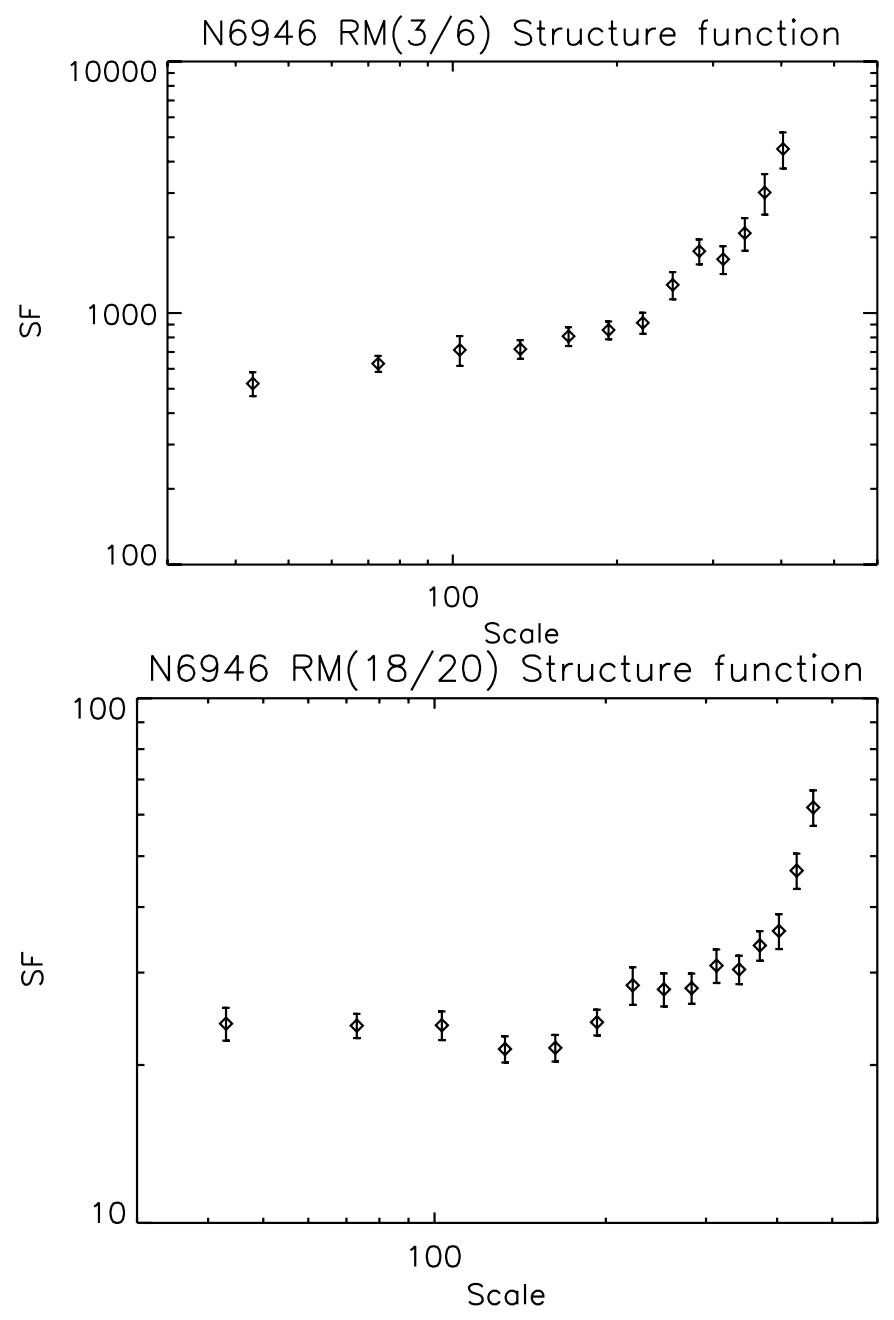

Fig. 13. Structure function (in logarithmic presentation) of the intrinsic Faraday rotation measures $R M_{i}$ between $\lambda 3.5 \mathrm{~cm}$ and $\lambda 6.2 \mathrm{~cm}$ (top) and between $\lambda 18 \mathrm{~cm}$ and $\lambda 20 \mathrm{~cm}$ (bottom) at $15^{\prime \prime}$ resolution. The scale is measured in arcseconds.

errors in $S F$ were computed from the standard deviations. The $S F$ of $R M_{i}$ between $\lambda 3.5 \mathrm{~cm}$ and $\lambda 6.2 \mathrm{~cm}$ is flat (slope $\simeq 0.3$ ) until 4' $(6 \mathrm{kpc})$ and then steepens (slopes of $\simeq 2-4)$. The $S F$ of $R M_{i}$ between $\lambda 18 \mathrm{~cm}$ and $\lambda 20 \mathrm{~cm}$ is very flat (slope $\simeq 0.0$ ) until $3^{\prime}$ $(5 \mathrm{kpc})$ and then mildly steepens (slope $\simeq 0.3$ ). Hence, Faraday effects shift spectral energy from large to small scales.

The $R M_{i}$ structures seen in NGC 6946 are correlated on scales of $\geq 6 \mathrm{kpc}$ which is due to the magnetic arms hosting coherent regular fields. On scales of $1-6 \mathrm{kpc}$ the $R M_{i}$ distribution is mostly chaotic, without indications of systematic structures in the coherent magnetic fields, like Parker loops. Coherent fields on kpc scales also exist in the Milky Way, measured from pulsar rotation measures (Han et al. 2006) and from Zeeman splitting of $\mathrm{OH}$ maser lines (Fish et al. 2003).

\subsection{Faraday depolarization}

The new polarization maps at $\lambda 18.0 \mathrm{~cm}$ and $\lambda 20.5 \mathrm{~cm}$ at $15^{\prime \prime}$ resolution (Fig. 6 bottom) do not show more polarized emission in the SW quadrant than the previous data at lower resolution (Beck 1991). Therefore 2D depolarization mechanisms acting in the sky plane, like Faraday dispersion in a foreground screen or $R M$ gradients, are less important in NGC 6946. 3D Faraday depolarization mechanism within the emitting volume, i.e. differential Faraday rotation or Faraday dispersion, dominate. In contrast to NGC 6946, the systematic pattern of depolarization in $\mathrm{M} 31$ shows that most of the depolarization in that galaxy is due to foreground $R M$ gradients. Compared to NGC 6946, RMs in M 31 are larger because the coherent regular field is stronger and the galaxy's inclination is higher (Fletcher et al. 2004).

Faraday depolarization is usually defined as the ratio $D P$ of the degrees of polarization synchrotron emission at two wavelengths. This requires subtraction of the thermal emission which is subject to uncertainties (see Sect. 3.2). Instead, $D P$ was computed in this paper by: $D P=\left(P I_{1} / P I_{2}\right) \times\left(v_{2} / v_{1}\right)^{\alpha_{\mathrm{n}}}$ where $\alpha_{\mathrm{n}}=-1.0$ is the synchrotron spectral index, assumed to be constant across the galaxy. Deviations in $\alpha_{\mathrm{n}}$ affect $D P$ less severely than the estimate of thermal fraction of the radio emission.

$D P(6 \mathrm{~cm} / 3 \mathrm{~cm})$ (Fig. 14 left) is around 1 in both main magnetic arms and in the inner galaxy east of the centre. Smaller values around 0.7 , i.e. stronger depolarization, are found $1-3^{\prime}$ south-west of the centre. $D P(20 \mathrm{~cm} / 6 \mathrm{~cm})$ (Fig. 14 right) varies over a much larger range. While $D P$ in the northern magnetic arm is around 0.4 , strong depolarization ( $D P$ between 0.1 and 0.2 ) occurs in the south-western quadrant.

Differential Faraday rotation within the emitting layer leads to depolarization which varies with $\lambda$ as a $\sin (x) / x$ function, with $x=2\left|R M_{i}\right| \lambda^{2}$ (Sokoloff et al. 1998). At $\lambda 20.5 \mathrm{~cm}$ strong $D P$ is expected for $\left|R M_{i}\right|>30 \mathrm{rad} \mathrm{m}^{-2}$, with many lines of zero polarization ("canals") along level lines with $\left|R M_{i}\right|=n 37.5 \mathrm{rad} \mathrm{m}^{-2}$. Only two canals were identified which are probably generated in the Galactic foreground (Sect. 3.10). Hence, differential Faraday rotation in NGC 6946 cannot explain the large-scale asymmetry in $D P$ (Sect. 4.2).

The intrinsic rotation measures between $\lambda 18.0 \mathrm{~cm}$ and $\lambda 20.5 \mathrm{~cm}$ are a factor of about five smaller than those between $\lambda 3.5 \mathrm{~cm}$ and $\lambda 6.2 \mathrm{~cm}$. The galaxy is not transparent to polarized decimeter radio waves, it is Faraday thick (Beck 1991; Ehle \& Beck 1993). Berkhuijsen et al. (1997) successfully modeled the similar situation in the spiral galaxy M 51 by assuming Faraday dispersion, which depolarizes most of the disk, plus a foreground layer in the upper disk or in the halo which have a lower polarized intensity and rotation measure than the full disk.

Internal Faraday dispersion by turbulence in the magnetoionic interstellar medium along the line of sight is the probable source of depolarization at long wavelengths. It is computed as (Sokoloff et al. 1998):

$p=p_{0}[1-\exp (-2 S)] /(2 S)$,

where $S=\sigma_{\mathrm{RM}}^{2} \lambda^{4} . \sigma_{\mathrm{RM}}$ is the dispersion in intrinsic rotation measure $R M_{i}$. (Note that a factor of two was missing in Beck 1991 and in Ehle \& Beck 1993.) $D P=0.2$ at $\lambda 20.5 \mathrm{~cm}$ needs a dispersion of $\sigma_{\mathrm{RM}} \simeq 38 \mathrm{rad} \mathrm{m}^{-2}$.

Faraday dispersion is an effect of the turbulent interstellar medium and can be written as $\sigma_{\mathrm{RM}}=0.81 n_{\mathrm{e}} B_{\mathrm{r}} d(L f / d)^{0.5}$ where $n_{\mathrm{e}}$ is the thermal electron density of the diffuse ionized gas (in $\mathrm{cm}^{-3}$ ), $B_{\mathrm{r}}$ the random field strength (in $\mu \mathrm{G}$ ), $L$ the pathlength through the thermal gas (in pc), $d$ the turbulent scale (in $\mathrm{pc}$ ), and $f$ the filling factor of the Faraday-rotating gas. Faraday rotation and depolarization occur in the diffuse ionized medium with a large filling factor, while the thermal optical and thermal radio emission is dominated by $\mathrm{H}_{\text {II }}$ regions with a small filling factor (see discussion in Ehle \& Beck 1993). Standard values of $n_{\mathrm{e}}=0.03 \mathrm{~cm}^{-3}, B_{\mathrm{r}}=10 \mu \mathrm{G}, L=1000 \mathrm{pc}, d=50 \mathrm{pc}$ and $f=0.5$ yield the required $\sigma_{\mathrm{RM}}$.

In the south-western quadrant $D P$ decreases to $<0.2$ (Fig. 14 right). If due to Faraday dispersion, an increase of $n_{\mathrm{e}}$ or $B_{\mathrm{r}}$ is 

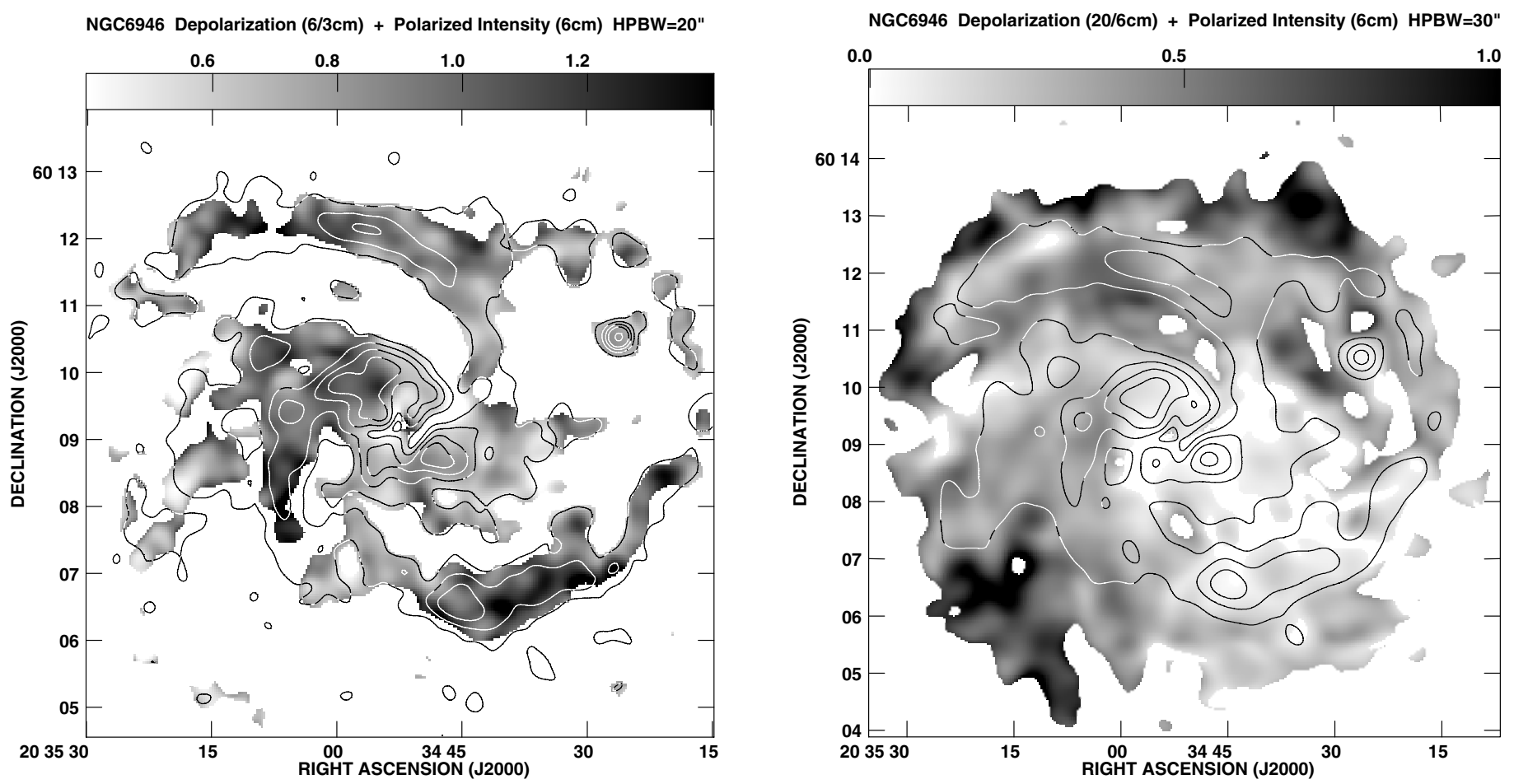

Fig. 14. Left: ratio between polarized intensities at $\lambda 6.2 \mathrm{~cm}$ and $\lambda 3.5 \mathrm{~cm}$ at $20^{\prime \prime}$ resolution, corrected for spectral index, as a measure of Faraday depolarization. The ratio was computed only at points where the polarized intensities at both wavelengths exceeds 4 times the rms noise (taken from that in $Q$ and $U$ ). The contours show the polarized intensity at $\lambda 6.2 \mathrm{~cm}$. The contour intervals are $1,2,3,4,6 \times 100 \mu \mathrm{Jy} / \mathrm{beam}$ area. Right: ratio between polarized intensities at $\lambda 20.5 \mathrm{~cm}$ and $\lambda 6.2 \mathrm{~cm}$ at $30^{\prime \prime}$ resolution, corrected for spectral index, as a measure of Faraday depolarization. The ratio was computed only at points where the polarized intensities at both wavelengths exceeds 4 times the rms noise. The contours show the polarized intensity at $\lambda 6.2 \mathrm{~cm}$. The contour intervals are $1,2,3,4 \times 200 \mu \mathrm{Jy} /$ beam area.

required, although star-formation activity and total radio emission is lower in this quadrant. The $D P$ asymmetry is probably an effect of field geometry (Sect. 4.2).

\subsection{Depolarization rings}

The $\lambda 20.5 \mathrm{~cm}$ polarization map shows three narrow rings of zero intensity (Fig. 15) with a $90^{\circ}$ jump of the polarization angle, resembling the canals in the $\lambda 21 \mathrm{~cm}$ and $\lambda 90 \mathrm{~cm}$ polarization maps of the Milky Way with resolutions of more than an arcminute (Gaensler et al. 2001; Uyanıker et al. 2003; Haverkorn et al. 2004) and in the $\lambda 20 \mathrm{~cm}$ polarization map of M 31 with $45^{\prime \prime}$ resolution (Shukurov \& Berkhuijsen 2003). These Galactic canals have a much longer angular extent than those in NGC 6946, most of them are not closed, and the few closed ones are irregular in shape.

The half-power widths ${ }^{4}$ of the rings in Fig. 15 are $4-6^{\prime \prime}$. The ring diameters are $20^{\prime \prime}$ (ring at RA, Dec $(2000)=20^{\mathrm{h}} 35^{\mathrm{m}} 10^{\mathrm{s}}$, $+60^{\circ} 12^{\prime} 35^{\prime \prime}$ ) and $12^{\prime \prime}$ (rings at RA, Dec $(2000)=20^{\mathrm{h}} 35^{\mathrm{m}} 02^{\mathrm{s}}$, $+60^{\circ} 12^{\prime} 50^{\prime \prime}$ and at $\left.20^{\mathrm{h}} 35^{\mathrm{m}} 10^{\mathrm{s}},+60^{\circ} 07^{\prime} 25^{\prime \prime}\right)$, corresponding to $530 \mathrm{pc}$ and $320 \mathrm{pc}$ if generated in NGC 6946, or to $0.1 \mathrm{pc} \times D$ and $0.06 \mathrm{pc} \times D$ at a distance $D$ (in kpc) in the Galactic foreground.

Depolarization canals or rings can be generated in the Milky Way by a superposition of diffuse background emission which is rotated in a Faraday screen with unrotated foreground emission (Sun et al. 2007). As our VLA synthesis observations were not

${ }^{4}$ Though structures in $Q$ or $U$ cannot be smaller than the beamsize, this is not true for maps of polarized intensity. Canals are the result of a sign change in the maps of Stokes $Q$ or $U$ or both (Fletcher \& Shukurov 2006). A steep gradient in Stokes $Q$ or $U$ may lead to a canal which is narrower than the beam. sensitive to the diffuse Galactic foreground emission, this explanation cannot be applied.

The foreground $R M_{\mathrm{fg}}$ in the Milky Way with a mean value of $40 \mathrm{rad} \mathrm{m}^{-2}$ can also generate a depolarization canal or ring if a jump or gradient of $\Delta R M_{\mathrm{fg}}=\left( \pm n+\frac{1}{2}\right) \pi / \lambda^{2}$ occurs on scales smaller than the beamsize (Fletcher \& Shukurov 2006). The rings of NGC 6946 could be signatures of small-scale structures in the magnetized Galactic interstellar medium with diameters of $d \simeq 0.06 \mathrm{pc} \times D$, smaller than those observed in Galactic surveys so far, in the size range of Galactic planetary nebulae or compact $\mathrm{H}$ II regions. Two of the rings have almost circular geometry. Assuming a spherical shape and a constant regular field strength of $2 \mu \mathrm{G}$ inside and outside the object, a $R M$ jump of $38 \mathrm{rad} \mathrm{m}^{-2}$ requires a mean electron density of $\left\langle n_{\mathrm{e}}\right\rangle \simeq 400 \mathrm{~cm}^{-3} / D$ where $D$ is the distance in kpc. With a $15^{\prime \prime}$ beam at $1.4 \mathrm{GHz}$, the thermal radio flux density per beam is $\simeq 0.6 \mu \mathrm{Jy} \times E M$ where $E M$ is the emission measure. With the numbers quoted above, $E M=\left\langle n_{\mathrm{e}}\right\rangle^{2} d / f \simeq 10^{4} \mathrm{~cm}^{-6} \mathrm{pc} / D / f$, where $f$ is the filling factor, yielding a large thermal flux density of several mJy/beam area, which is not detected in Fig. 2, so that the rings cannot originate in the Galactic foreground.

Shukurov \& Berkhuijsen (2003) and Fletcher \& Shukurov (2006) discussed that the canals can also be generated by internal differential Faraday rotation within the emitting medium. Such canals appear at $\lambda 20.5 \mathrm{~cm}$ at level lines of $R M_{i}= \pm n \pi /\left(2 \lambda^{2}\right)=$ $\pm n 37.5 \mathrm{rad} \mathrm{m}^{-2}$ and at $\lambda 18.0 \mathrm{~cm}$ at $\pm n 48.4 \mathrm{rad} \mathrm{m}^{-2}$ (Fletcher \& Shukurov 2006). (Note that $R M_{i}$ is the observed internal rotation measure which is half of the $R M_{0}$ through the whole source.) The intrinsic rotation measure which is relevant for internal differential Faraday rotation is that of the visible layer, i.e. the intrinsic rotation measure between $\lambda 18.0 \mathrm{~cm}$ and $\lambda 20.5 \mathrm{~cm}$ 

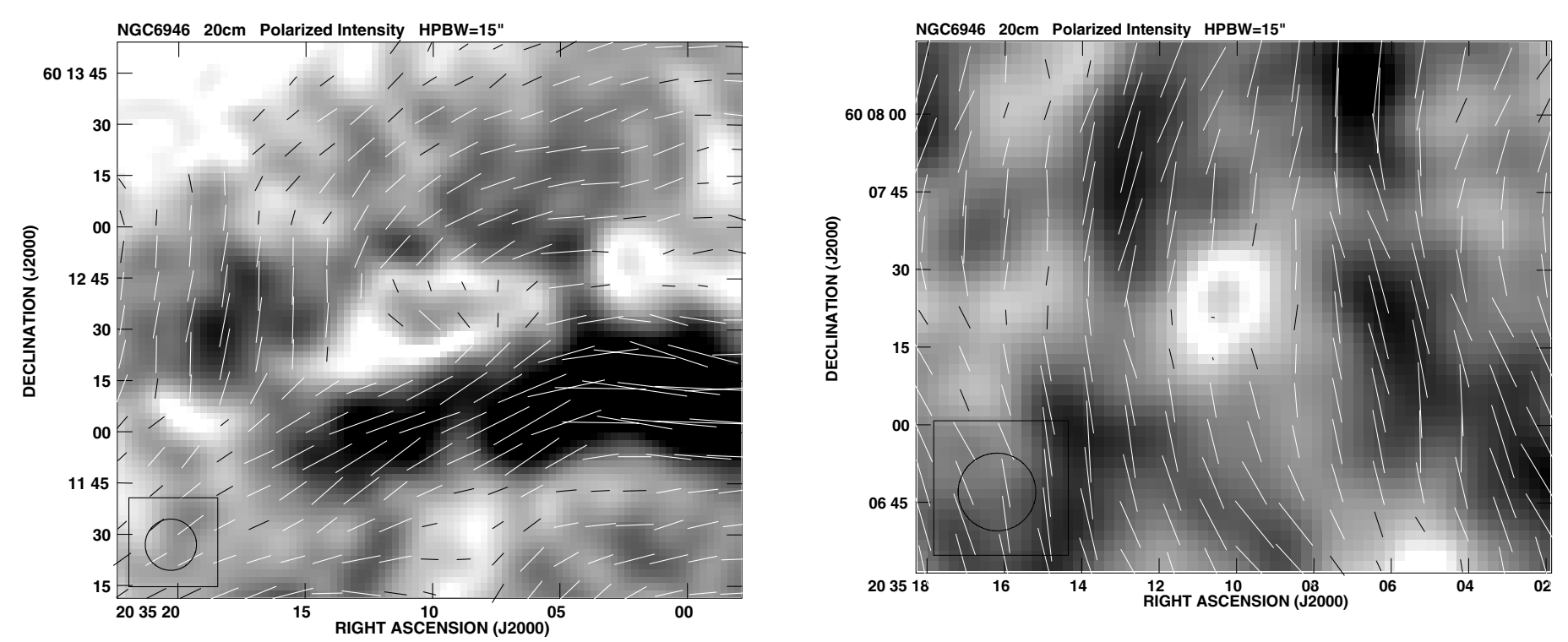

Fig. 15. Polarized intensity and observed $E$-vectors at $\lambda 20.5 \mathrm{~cm}$ at $15^{\prime \prime}$ resolution in the regions showing ring-like "canals".

(Fig. 12 right). The $R M_{i}$ values around the rings are smaller than the critical values for total internal depolarization, so that a steep increase towards the rings is required. Assuming a diameter $d \simeq 300 \mathrm{pc}$ (e.g. a H II complex), a spherical shape and a constant regular field strength of $3 \mu \mathrm{G}$ inside and outside the object, a $R M$ jump of $38 \mathrm{rad} \mathrm{m}^{-2}$ requires a mean electron density of $\left\langle n_{\mathrm{e}}\right\rangle \simeq 0.1 \mathrm{~cm}^{-3}$. The corresponding emission measure of $E M \simeq 3 \mathrm{~cm}^{-6} \mathrm{pc} / f$ yields a thermal flux density of $\simeq 40 \mu \mathrm{Jy} /$ beam area for $f=0.05$, only 2 times the rms noise at $\lambda 20.5 \mathrm{~cm}$ and hence not visible in Fig. 2.

\section{Discussion}

\subsection{The origin of the magnetic spiral arms}

The two bright inner magnetic spiral arms of NGC 6946 are not the effect of reduced depolarization in the regions between the optical spiral arms. Firstly, wavelength-dependent Faraday depolarization is not weaker (indicated by larger $D P$ values) in the magnetic arms (Fig. 14 right). Secondly, reduced wavelengthindependent depolarization (less tangled fields or less turbulent fields) can be excluded by the fact that the magnetic arms are seen also in total intensity (Figs. 1 and 2 right). The intensity enhancement in the magnetic arms above the background is about the same in polarized and in total intensity. Hence, the magnetic arms are real features where the strengths of the regular and of the total field are enhanced. After subtraction of the smooth, unpolarized disk emission, the degree of polarization is very high in the magnetic arms, reaching the maximum possible value of $75 \%$ in the northern magnetic arm, so that the magnetic field is totally aligned there.

Magnetic arms exist in several other gas-rich spiral galaxies of Hubble type Sc, for example in IC 342 (Krause 1993; Beck 2005), NGC 2997 (Han et al. 1999) and M 83 (Beck 2005), but are less prominent than in the classical case NGC 6946. In galaxies with strong density waves, like M 51, bright ridges at the inner edges of the spiral arms dominate the maps of polarized intensity (Patrikeev et al. 2006), but the diffuse polarized emission in the interarm regions may have a similar origin as the magnetic arms. Sb-type galaxies like M 31 and M 81 and flocculent galaxies like M 33 do not host magnetic arms (see Beck 2005 for a review). Hence, the phenomenon of magnetic arms seems to occur preferably in galaxies with a large mass of cold gas, a high star-formation rate and strong magnetic fields.

The analysis technique with $1 \mathrm{D}$ wavelet functions by Frick et al. (2000) is a tool to measure the pitch angle of spiral arm structures. Application to the NGC 6946 data indicated that the magnetic arms seen in polarization are phase-shifted "images" of the gas/optical arms, receding in the sense of galactic rotation, and hence could be the result of interaction between the gaseous density wave and the magnetic dynamo wave. The structure of the magnetic arms is more regular than that of the gas arms. Gas inside the corotation radius of $\simeq 10 \mathrm{kpc}$ (Rohde et al. 1999) rotates faster than the spiral pattern and crosses the magnetic arm before entering the gas arm, so that the magnetic arm is generated first. The properties of the gas arm could be determined by the magnetic arm.

Slow $2 D$ MHD waves in a galactic disk provide the required phase shift between the amplitudes of the gas and the magnetic field and also explain the alignment of the pitch angles of the magnetic field (Fan \& Lou 1996, 1997; Lou \& Fan 1998). However, magnetic arms are observed only in the differentially rotating part of NGC 6946 while in the model slow MHD waves exist only in the rigidly rotating part. Furthermore, slow MHD waves are unstable in three dimensions as they evolve into the modes of the Parker instabilities (Foglizzo \& Tagger 1995; Shukurov 2005).

Linear models of the turbulent galactic dynamo are in principle able to explain the two bright magnetic arms if dynamo action in the interarm regions is enhanced by increased magnetic diffusivity or a larger correlation length (Moss 1998; Shukurov 1998; Rohde et al. 1999). The $m=0$ azimuthal dynamo mode generates an axisymmetric spiral field with constant field direction, the $m=2$ azimuthal mode four magnetic spiral arms with alternating field directions. If the $m=0$ and $m=2$ azimuthal modes are superimposed with the same amplitudes, two arms remain which have the same direction of the radial field component with respect to the galaxy's centre, in agreement with the large-scale $R M$ distribution observed in NGC 6946 (Sect. 3.7). However, the dynamo model predicts that the phase shift between the magnetic arm and the optical arm varies with galactocentric radius and vanishes at the corotation radius, while the 
NGC6946 Magnetic Field Pitch Angles + Polarized Intensity (6cm) HPBW=20"

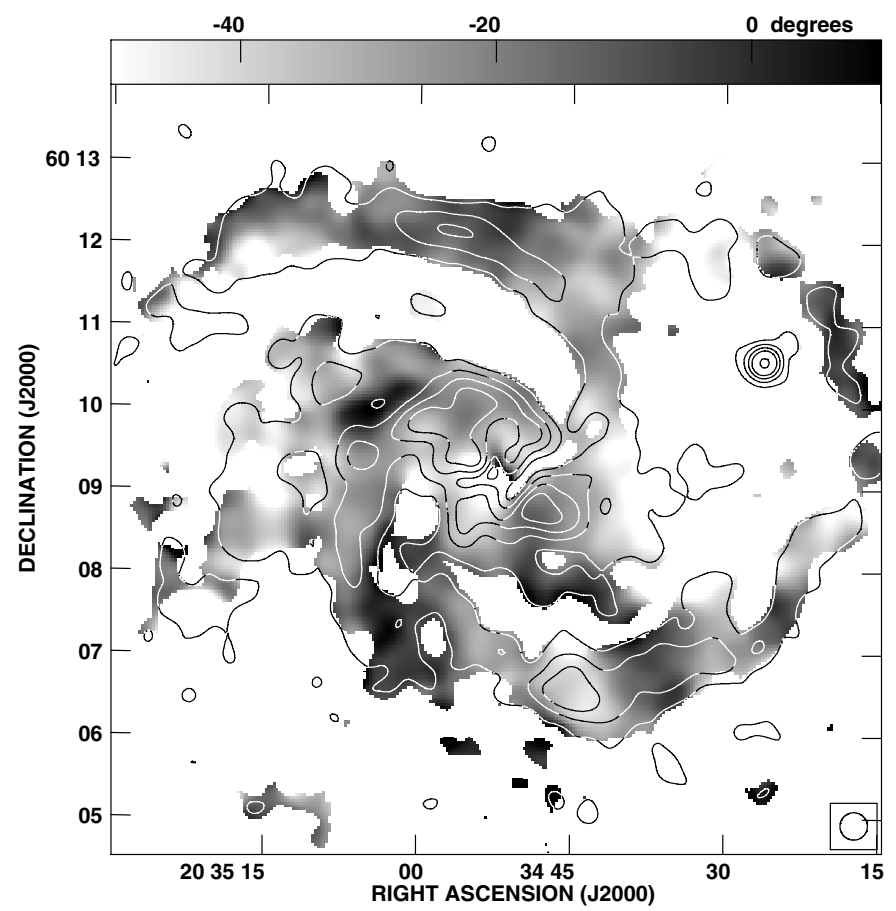

Fig. 16. Pitch angles of the magnetic field vectors at $20^{\prime \prime}$ resolution and contours of polarized intensity at $\lambda 6.2 \mathrm{~cm}$.

observed phase shift is almost constant with radius (Frick et al. 2000).

In the interpretation of the magnetic arms as dynamo modes, the pitch angle of the field vector depends on the dynamo numbers which reflect the physical conditions required for dynamo excitation (Shukurov 2000). If these conditions vary locally, the pitch angle will also vary. Rohde et al. (1999) showed that the average absolute field pitch angle in NGC 6946 is indeed smaller by about $10^{\circ}$ in the magnetic arms than in the nearby optical arms.

To quantify the regularity of the spiral field pattern in NGC 6946, field pitch angles were computed from the observed $B$-vectors. At each point of the map with sufficiently high polarized intensity, the position angle of the circumferential vector in the plane of galaxy, projected into the sky plane, was subtracted from the position angle of the Faraday-corrected $B$-vectors (Fig. 6 top right). The plane of the galaxy was assumed to be inclined by $38^{\circ}$ to the sky plane. The major axis of the galaxy plane projected to the sky plane was assumed to have a position angle of $60^{\circ}$ (Carignan et al. 1990). Figure 16 shows the resulting map of the pitch angles of the magnetic field vectors in the sky plane. The average pitch angle of the field vectors in the northern magnetic arm is $-20^{\circ} \pm 10^{\circ}$, with a tendency of decreasing absolute values from the inner to the outer part. The rms variations are consistent with those expected from rms noise in the original maps. The field in the southern main magnetic arm shows larger variations in pitch angle. In its inner part (east of the galaxy centre) the field's pitch angle is $-25^{\circ} \pm 7^{\circ}$, while the outer part (south to west) the pitch angle varies between $0^{\circ}$ (azimuthal) and $-40^{\circ}$ in a quasi-periodical manner on a length scale of about $2^{\prime}(3 \mathrm{kpc})$, where the structure function (Fig. 13 top) starts to rise.

Comparison of the pitch angles of the magnetic field vectors (Fig. 16) with the Faraday rotation measures (Fig. 12 left) reveals some degree of correlation. For example, $R M$ values of $\leq-100 \mathrm{rad} \mathrm{m}^{-2}$ in the south-east and at the western edge of the map correspond to small absolute pitch angles in Fig. 16. Vice versa, $R M \geq 100 \mathrm{rad} \mathrm{m}^{-2}$ about $3^{\prime}$ west and east of the centre correspond to large absolute pitch angles (about $-40^{\circ}$ ). Crosscorrelation of the pitch angles of Fig. 16 with the $R M$ data between $\lambda 3.5 \mathrm{~cm}$ and $\lambda 6.2 \mathrm{~cm}$ at the same resolution yields a negative slope of -0.32 and a correlation coefficient of -0.45 . With more than 50 independent points and a Student- $t$ value of 4 this is a significant correlation. As $R M$ shows the regular field component along the line of sight and the pitch angle the field projection in the sky plane, systematic 3D deviations of the magnetic field lines from the plane of the galaxy are the probable cause for the correlated deviations, such as helical fields (see Sect. 4.2).

Although the field pitch angles are not constant in NGC 6946, they may still be aligned with the spiral structures. To measure the pitch angles of the spiral structures the wavelet technique of Frick et al. (2000) was applied to the polarized intensity map (Fig. 6 top right). The mean pitch angle of the northern magnetic arm structure is $-25^{\circ} \pm 1^{\circ}$ and that of the southern magnetic arm is $-31^{\circ} \pm 2^{\circ}$ (inner part, from east to south) and $-15^{\circ} \pm 1^{\circ}$ (outer part, from south to west). Hence, the pitch angle of the magnetic field vectors and that of the structure in polarized intensity agree well in the northern arm, but only in parts of the southern arm. In M 51 the magnetic field in one polarized arm has the same pitch angle (within a few degrees) as the arm itself, but the field's pitch angle is systematically smaller by $10-20^{\circ}$ than the pitch angle of the other polarized arm (Patrikeev et al. 2006).

Alignment of the field vectors along the spiral structure can also be achieved by compression of field and gas, e.g. in density waves. However, the alignment of the field along the magnetic arms of NGC 6946 cannot be explained by compression because any sign of gas compression is missing. Density waves are relatively weak in NGC 6946, probably due to the lack of a companion galaxy and of a massive bar. Only in the inner region, at the inner edge of the inner northern optical arm, density waves are sufficiently strong for significant compression and field alignment along the optical arm (Sect. 3.6).

The new observations presented in this paper raise challenges to turbulent dynamo models. Firstly, two main magnetic arms in NGC 6946 are only the tip of the iceberg, the brightest parts of a network of magnetic features (Figs. 7 and 17). The outer northern magnetic arms are also located between $\mathrm{H}$ I arms. A dynamo origin of these outer arms is possible by the excitation of azimuthal modes higher than $m=2$, but needs to be tested with Faraday rotation measures, which however is not possible with the present data. At wavelengths of $\lambda 18 \mathrm{~cm}$ and larger, the galaxy is no longer transparent to polarized radio waves from the disk, so that no reliable $R M$ can be determined. At shorter wavelengths, the sensitivity of present-day radio telescopes is insufficient to detect polarized synchrotron emission from the outer magnetic arms. Polarized background sources of sufficiently small angular size do not suffer from Faraday depolarization and can be used to determine $R M s$ within the galaxy. With present-day telescopes the number density of polarized sources is too low. Future measurements at long wavelengths are favorable (e.g. with LOFAR) yielding a smaller $R M$ error.

Secondly, the total alignment of the field in the northern bright magnetic arm is hard to understand because turbulent dynamos always generate small-scale random fields simultaneously to large-scale fields. Either the production of random fields is suppressed in the magnetic arms, or these random fields are distributed smoothly over the whole disk, observable as smooth, 


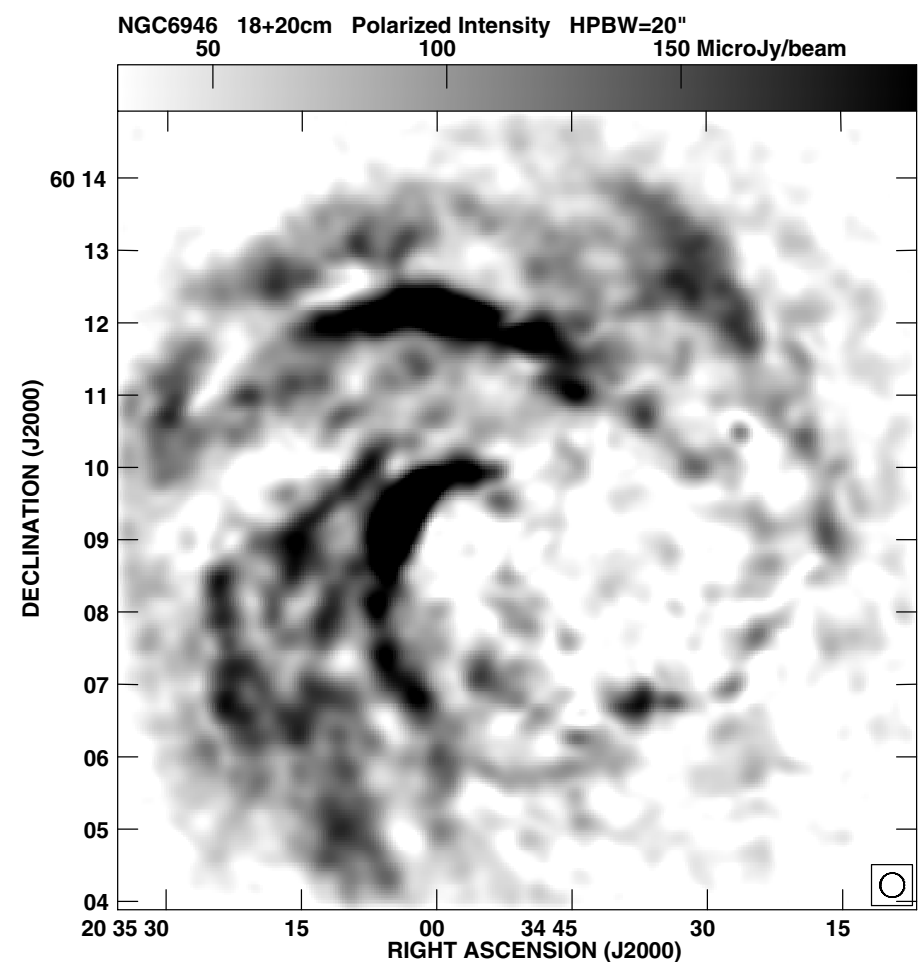

Fig. 17. Average polarized intensity at $\lambda 18.0 \mathrm{~cm}$ and $\lambda 20.5 \mathrm{~cm}$ at $20^{\prime \prime}$ resolution.

unpolarized emission. Future turbulent dynamo models including the simultaneous generation of small-scale and large-scale magnetic fields are required.

Thirdly, the partial alignment of the field along the spiral structures and the network of magnetic arms call for nonlinear dynamos. In linear dynamo models, the pitch angle of the magnetic field vectors and that of the generated polarized spiral arm structure are independent. In NGC 6946 and several other spiral galaxies, these two pitch angles are mostly similar. Nonlinear dynamo models are required where the field can back-react onto the gas flow.

The comparison of energy densities in Sect. 3.3 demonstrated that the magnetic fields control the flow of the ionized gas and are of similar importance as the turbulent motions of the neutral gas, possibly even dominating (Fig. 5). An influence of the magnetic field onto the gas spiral arms is well possible.

A few steps steps have already been done along this way. Elstner et al. (2000) modelled the magnetic field in a spiral galaxy from dynamo action and shear in a time-dependent gas flow, without back-reaction onto the gas flow. The regular magnetic field was shown to avoid the gas arms, forming magnetic arms, as observed, and the spiral arm flow adjusts the field along the regions of high gas density. However, the pitch angles of the magnetic field vectors are too small in the interarm regions. Inclusion of the magneto-rotational instability (MRI) as the main source of turbulence in the outer parts of galaxies (Sellwood \& Balbus 1999; Dziourkevitch et al. 2004) provide relatively strong seed fields which may enhance dynamo action and generate mixed dynamo modes with magnetic arms. The model by Nishikori et al. (2006) includes MRI, but neglects spiral flows, dynamo action and the input of turbulent energy by supernovae. Many magnetic arms appear in their model, but with frequent reversals of the azimuthal field direction along radius which are not observed. Furthermore, the field also reverses from the disk to the halo, so that the $R M$ of polarized background sources

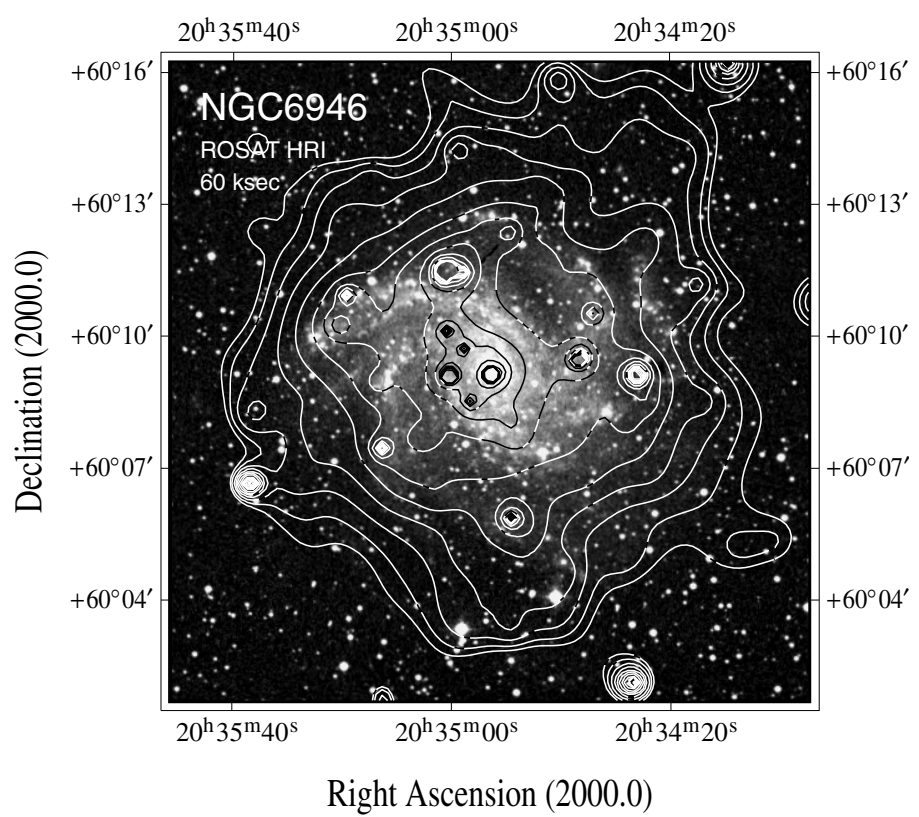

Fig. 18. Deep 60 ks ROSAT HRI image of NGC 6946 (Ehle, priv. comm.) overlaid onto an optical image.

should be small, which also is in conflict with the observations presented in this paper.

Future MHD models should include the detailed gas flow (as obtained from high-resolution $\mathrm{H}_{\mathrm{I}}$ observations) and allow for back-reaction of the generated magnetic field onto the gas flow.

\subsection{The origin of the depolarization asymmetry}

The second unexpected discovery from radio polarization observations of NGC 6946 was the large-scale asymmetry in Faraday depolarization. The strongest depolarization occurs in the SW quadrant (Fig. 14). An origin in the foreground of the Milky Way by strong RM gradients can be excluded because the RM foreground is smooth (Sect. 3.10). Strong Faraday dispersion in the Galactic foreground in a region of about $5^{\prime}$ size is possible but improbable. Faraday rotation at long wavelengths is also enhanced in the SW quadrant (Beck 1991) which would cause enhanced depolarization only if both phenomena are located in the emitting volume within NGC 6946. Enhanced depolarization by enhanced thermal density or field strength in the disk of NGC 6946 is improble as the star formation rate is low in this part of the galaxy. The remaining explanations are halo fields or field geometry.

Faraday depolarization is generated by the field components along the line of sight. The required difference between the vertical field components on the eastern and western major axis cannot be achieved by any symmetric field configuration. The asymmetry can be interpreted as the effect of a vertical magnetic field (Beck 1991). This phenomenon may resemble solar coronal holes generated by fast streams of hot gas above regions of low solar activity. In the centre of this region a huge stellar complex with high wind speed is observed (Efremov et al. 2002; Larsen et al. 2002). If this is the origin of strong outflows of hot gas, enhanced X-ray emission should be observable from the halo in the SW quadrant of NGC 6946. Figure 18 shows a deep ROSAT image which reveals diffuse, extended emission around the galaxy. The extension of the X-ray emission is larger towards the south and towards the north-west, but there is no significant 
Table 2. Magnetic phenomena in NGC 6946 and their probable origins.

\begin{tabular}{ll}
\hline \hline Total magnetic energy density $\simeq$ turbulent energy density in inner galaxy & Field amplification by turbulent gas flows \\
Total magnetic energy density dominates in outer galaxy & Magneto-rotational instability ? \\
Low degrees of polarization in gas spiral arms & Field tangling due to star-formation activity \\
Large-scale pattern in Faraday rotation & Coherent fields \\
Magnetic arms with regular, almost completely aligned fields & Efficient dynamo action in interarm regions \\
Two inner magnetic arms & Superposition of dynamo modes $m=0$ and $m=2$ \\
No large-scale field reversals & Higher dynamo modes weak or absent in inner galaxy \\
Outer magnetic arms & Higher dynamo modes or shear flows in outer galaxy \\
Alignment of ordered fields along spiral structure & Interaction between gas flow and magnetic field \\
Magnetic arms are phase-shifted images of gas arms & Magnetic field may trigger the formation of gas spiral arms \\
North-south asymmetry in Faraday rotation & Spiral field points inwards in both inner magnetic arms \\
Enhanced magnetic field along inner edge of inner gas spiral arm & Compression or shear by density waves \\
Smooth turning of field lines into inner gas spiral arm & No shock \\
Mild field compression in inner gas spiral arm & Magnetic field connected to warm, diffuse gas \\
Anti-correlation of $R M$ and field pitch angle & Helical fields \\
$R M$ structure function flat until $6 \mathrm{kpc}$ scale & Uncorrelated field structures below $6 \mathrm{kpc}$ scale \\
$R M$ structure function rising for scales larger than $6 \mathrm{kpc}$ & Large-scale coherent fields \\
Depolarization rings at $\lambda 20 \mathrm{~cm}$ & Differential Faraday rotation in H $\mathrm{H}$ complexes \\
Strong Faraday depolarization at $\lambda 18 \mathrm{~cm}$ and $\lambda 20 \mathrm{~cm}$ & Faraday dispersion by random fields \\
Large-scale asymmetry in Faraday depolarization & Helical fields ? \\
\hline
\end{tabular}

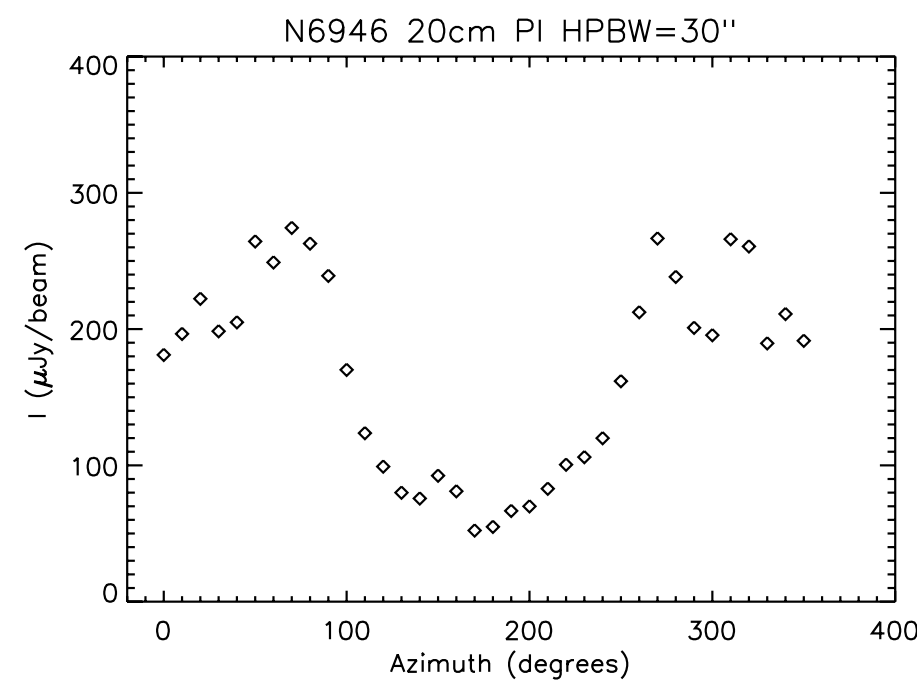

Fig. 19. Polarized intensity at $\lambda 20.5 \mathrm{~cm}$ at $30^{\prime \prime}$ resolution, averaged in sectors of $10^{\circ}$ width in azimuthal angle in the galaxy plane and between $1 \mathrm{kpc}$ and $10 \mathrm{kpc}$ radius from the centre of NGC 6946. The azimuthal angle is counted counterclockwise from the galaxy's northeastern major axis (at $60^{\circ}$ position angle). Errors due to rms noise are $\simeq 5 \mu \mathrm{Jy} /$ beam area, smaller than the symbol sizes.

increase in the region of strong depolarization. More sensitive XMM data are forthcoming (Ehle \& Beck, in prep).

A large-scale field asymmetry could also be caused by a bisymmetric $(m=1)$ dynamo mode which dominates in the halo. However, Ehle \& Beck (1993) demonstrated that the rotation measures between $\lambda 20.5 \mathrm{~cm}$ and $\lambda 2.8 \mathrm{~cm}$ (tracing disk plus halo) can be fitted by a single-periodic sine wave of similar phase than the RM between $\lambda 6.3 \mathrm{~cm}$ and $\lambda 2.8 \mathrm{~cm}$ (tracing the disk only), excluding the presence of a bisymmetric mode with an amplitude similar to those of the $m=0$ and $m=2$ modes.

Interestingly, depolarization asymmetries exist in several galaxies. The polarized intensity of NGC 6946 at $\lambda 20 \mathrm{~cm}$ has a broad minimum centered on the south-western major axis (at $180^{\circ}$ azimuth in Fig. 19). The same phenomenon is observed in M 83 which has a similar inclination and orientation in space as NGC 6946 (Neininger et al. 1993). In IC 342 the minimum at $\lambda 20 \mathrm{~cm}$ is also centered on the major axis, but on the northeastern side (Krause et al. 1989). Hence, all models of asymmetric vertical fields are improbable because the field geometry cannot depend on the direction to the observer. Taking into account that the sense of winding of the spiral arms in IC 342 is reversed compared to NGC 6946 and M 83, a common origin as an effect of the geometry of the field with respect to the observer is required. A possible explanation are helical fields of sufficiently large diameter, so that Faraday depolarization of emission from the far side of the helix is different from that from the near side (Urbanik et al. 1997). Detailed models are in preparation.

\section{Summary: NGC 6946, a gold mine of magnetic phenomena}

NGC 6946 is the ideal object to study magnetic fields with radio polarization. Its size is large enough to allow high spatial resolution, and not too large, so that it can be covered by a one single pointing with the VLA at wavelengths larger than $\lambda 6 \mathrm{~cm}$. NGC 6946 has one of the highest star-formation rates among spiral galaxies and one of the strongest magnetic fields. It is no surprise that this is the only spiral galaxy so far which has been observed in radio polarization at five frequencies with high resolution and high sensitivity.

The results on magnetic field properties in NGC 6946 and their probable origins are summarized in Table 2. The wealth of different phenomena requires a combination of physical origins, like dynamo action, density waves and shear flows. These phenomena occur also in other galaxies, but with different weighting: Polarized radio emission from M 31 is dominated by its coherent dynamo field of axisymmetric type; the strong density waves of M 51 generate a dominating anisotropic field by compression; the anisotropic fields of the barred galaxies NGC 1097 and NGC 1365 are due to compression and shear; the Large Magellanic clouds and the Magellanic-type galaxy NGC 4449 hosts weak coherent dynamo fields, and small irregular galaxies show turbulent fields only without signs of coherency (see Beck 2005, for a review). NGC 6946 offers everything. 
The low-frequency radio telescope LOFAR is under construction and will map the magnetic fields in the outer disks of galaxies which are populated by low-energy cosmic-ray electrons. The sensitivity of the extended VLA (EVLA) will allow deeper mapping of galaxies in polarization and Faraday rotation. Finally, the planned Square Kilometre Array (SKA) will allow to search for magnetic fields in distant galaxies and to accurately measure the spectrum of dynamo modes in nearby galaxies (Beck 2006). With the SKA the number density of polarized sources on sky will be increase by several orders of magnitude and allow to measure a dense grid of $R M s$ through galaxies (Beck \& Gaensler 2004). Thanks to its large angular extent, NGC 6946 will be one of the prime targets for these forthcoming radio telescopes.

Acknowledgements. I wish to thank Elly M. Berkhuijsen, Andrew Fletcher and especially Anvar Shukurov for useful discussions and many helpful comments. I thank Bülent Uyanıker and Tom Pannuti for reducing the Effelsberg $\lambda 11 \mathrm{~cm}$ and $\lambda 21 \mathrm{~cm}$ observations, Matthias Ehle for providing his ROSAT map, and Bülent Uyanıker for his software to compute structure functions.

\section{References}

de Avillez, M. A., \& Breitschwerdt, D. 2005, A\&A, 436, 585

Battaner, E., \& Florido, E. 2000, Fund. Cosm. Phys., 21, 1

Battaner, E., \& Florido, E. 2007, AN, 328, 92

Beck, R. 1991, A\&A, 251, 15

Beck, R. 2001, Space Sci. Rev., 99, 243

Beck, R. 2005, in Cosmic Magnetic Fields, ed. R. Wielebinski, \& R. Beck (Berlin: Springer), 41

Beck, R. 2006, AN, 327, 512

Beck, R., \& Golla, G. 1988, A\&A, 191, L9

Beck, R., \& Hoernes, P. 1996, Nature, 379, 47

Beck, R., \& Gaensler, B. 2004, New Astr. Rev., 48, 1289

Beck, R., \& Krause, M. 2005, AN, 326, 414

Beck, R., Brandenburg, A., Moss, D., Shukurov, A., \& Sokoloff, D. 1996, ARA\&A, 34, 155

Beck, R., Shoutenkov, V., Ehle, M., et al. 2002, A\&A, 391, 83

Beck, R., Shukurov, A., Sokoloff, D., \& Wielebinski, R. 2003, A\&A, 411, 99

Beck, R., Fletcher, A., Shukurov, A., et al. 2005, A\&A, 444, 739

Belyanin, M., Sokoloff, D., \& Shukurov, A. 1993, Geophys. Astrophys. Fluid Dyn., 68, 227

Berkhuijsen, E. M., Horellou, C., Krause, M., et al. 1997, A\&A, 318, 700

Berkhuijsen, E. M., Beck, R., \& Hoernes, P. 2003, A\&A, 398, 937

Bicay, M. D., Helou, G., \& Condon, J. J. 1989, ApJ, 338, L53

Boomsma, R., Oosterloo, T. A., Fraternali, F., van der Hulst, J. M., \& Sancisi, R. 2005, in Extraplanar Gas, ed. R. Braun, ASP Conf. Proc., 331, 247

Boulares, A., \& Cox, D. P. 1990, ApJ, 365, 544

Braun, R., Oosterloo, T. A., Morganti, R., Klein, U., \& Beck, R. 2007, A\&A, 461,455

Breitschwerdt, D., Dogiel, V. A., \& Völk, H. J. 2002, A\&A, 385, 216

Burn, J. 1966, MNRAS, 153, 67

Carignan, C., Charbonneau, P., Boulanger, F., \& Viallefond, F. 1990, A\&A, 234, 43

Condon, J. J., Cotton, W. D., Greisen, E. W., et al. 1998, AJ, 115, 1693

Cox, D. P. 2005, ARA\&A, 43, 337

Dale, D. A., Silberman, N. A., Helou, G., et al. 2000, AJ, 120,583

Dziourkevitch, N., Elstner, D., \& Rüdiger, G. 2004, A\&A, 423, L29

Ehle, M., \& Beck, R. 1993, A\&A, 273, 45

Efremov, Y. N., Pustilnik, S. A., Kniazev, A. Y., et al. 2002, A\&A, 389, 855

Elstner, D., Otmianowska-Mazur, K., von Linden, S., \& Urbanik, M. 2000, A\&A, 357, 129

Fan, Z., \& Lou, Y.-Q. 1996, Nat, 383, 800

Fan, Z., \& Lou, Y.-Q. 1997, MNRAS, 291, 91

Ferguson, A. M. N., Wyse, R. F. G., Gallagher, J. S., \& Hunter, D. A. 1998, ApJ, 506, L19
Fish, V. L., Reid, M. J., Argon, A. L., \& Menten, K. M. 2003, ApJ, 596, 328 Fletcher, A., \& Shukurov, A. 2001, MNRAS, 325, 312

Fletcher, A., \& Shukurov, A. 2006, MNRAS, 371, L21

Fletcher, A., Berkhuijsen, E. M., Beck, R., \& Shukurov, A. 2004, A\&A, 414, 53

Fletcher, A., Beck, R., Berkhuijsen, E. M., Horellou, C., \& Shukurov, A. 2007, A\&A, in prep.

Foglizzo, T., \& Tagger, M. 1995, A\&A, 301, 293

Frick, P., Beck, R., Shukurov, A., et al. 2000, MNRAS, 318, 925

Frick, P., Beck, R., Berkhuijsen, E. M., \& Patrikeev, I. 2001, MNRAS, 327, 1145

Gaensler, B. M., Dickey, J. D., McClure-Griffiths, N. M., et al. 2001, ApJ, 549, 959

Gómez, G. C., \& Cox, D. P. 2002, ApJ, 580, 235

Green, D. A. 2001, in High Energy Gamma-Ray Astronomy, ed. F. A. Aharonian, \& H. J. Völk (New York: Melville), 59

Han, J. L., Beck, R., Ehle, M., Haynes, R. F., \& Wielebinski, R. 1999, A\&A, 348,405

Han, J. L., Manchester, R. N., Lyne, A. G., Qiao, G. J., \& van Straten, W. 2006, ApJ, 642, 868

Haverkorn, M., Katgert, P., \& de Bruyn, A. G. 2004, A\&A, 427, 459

Haverkorn, M., Gaensler, B. M., Brown, J. C., et al. 2006, ApJ, 637, L33

Heiles, C., \& Crutcher, R. 2005, in Cosmic Magnetic Fields, ed. R. Wielebinski, \& R. Beck (Berlin: Springer), 137

Hoernes, P., Berkhuijsen, E. M., \& Xu, C. 1998, A\&A, 334, 57

Kalberla, P. M. W., \& Kerp, J. 1998, A\&A, 339, 745

Kamphuis, J., \& Sancisi, R. 1993, A\&A, 273, L31

Kim, W.-T., \& Ostriker, E. C. 2002, ApJ, 570, 132

Klein, U., Beck, R., Buczilowski, U. R., \& Wielebinski, R. 1982, A\&A, 108, 176

Krause, F., \& Beck, R. 1998, A\&A, 335, 789

Krause, M. 1993, in The Cosmic Dynamo, ed. F. Krause et al. (Dordrecht: Kluwer), 305

Krause, M., Hummel, E., \& Beck, R. 1989, A\&A, 217, 4

Larsen, S. S., Efremov, Y. N., Elmegreen, B. G., et al. 2002, ApJ, 567, 896

Lou, Y.-Q., \& Fan, Z. 1998, ApJ, 493, 102

Mac Low, M.-M., \& Klessen, R. S. 2004, Rev. Mod. Phys., 76, 125

Mac Low, M.-M., Balsara, D. S., Kim, J., \& de Avillez, M. A. 2005, ApJ, 626, 864

Moss, D. 1998, MNRAS, 297, 860

Murphy, E. J., Helou, G., Braun, R., et al. 2006, ApJ, 651, L111

Neininger, N., Beck, R., Sukumar, S., \& Allen, R. J. 1993, A\&A, 274, 687

Niklas, S., \& Beck, R. 1997, A\&A, 320, 54

Nishikori, H., Machida, M., \& Matsumoto, R. 2006, ApJ, 641, 862

Harnett, J. I., Beck, R., \& Buczilowski, U. R. 1989, A\&A, 208, 32

Patrikeev, I., Fletcher, A., Stepanov, R., et al. 2006, A\&A, 458, 441

Priklonsky, V. I., Shukurov, A., Sokoloff, D., \& Soward, A. 2000, Geophys. Astrophys. Fluid Dyn., 93, 97

Rohde, R., Beck, R., \& Elstner, D. 1999, A\&A, 350, 423

Schinnerer, E., Böker, T., Emsellem, E., \& Lisenfeld, U. 2006, ApJ, 649, 181

Sellwood, J. A., \& Balbus, S. A. 1999, ApJ, 511, 660

Shukurov, A. 1998, MNRAS, 299, L21

Shukurov, A. 2000, in The Interstellar Medium in M 31 and M 33, ed. E. M. Berkhuijsen et al., 191

Shukurov, A. 2005, in Cosmic Magnetic Fields, ed. R. Wielebinski, \& R. Beck (Berlin: Springer), 129

Shukurov, A., \& Berkhuijsen, E. M. 2003, MNRAS, 342, 496, and MNRAS, 345, 1392, Erratum

Silk, J., \& Langer, M. 2006, MNRAS, 371, 444

Sokoloff, D. D., Bykov, A. A., Shukurov, A., et al. 1998, MNRAS, 299, 189, and MNRAS, 303, 207, Erratum

Strong, A. W., \& Mattox, J. R. 1996, A\&A, 308, L21

Sun, X. H., Han, J. L., Reich, W., et al. 2007, A\&A, 463, 993

Tabatabaei, F., Beck, R., Krügel, E., et al. 2007, A\&A, in prep.

Tully, R. B. 1988, Nearby Galaxies Catalog (Cambridge: Cambridge University Press)

Urbanik, M., Elstner, D., \& Beck, R. 1997, A\&A, 326, 465

Uyanıker, B., Landecker, T. L., Gray, A. D., \& Kothes, R. 2003, ApJ, 585, 785

Vogt, C., \& Enßlin, T. A. 2003, A\&A, 412, 373

Vollmer, B., Soida, M., Beck, R., et al. 2007, A\&A, 464, L37

Walsh, W., Beck, R., Thuma, G., et al. 2002, A\&A, 388, 7 